\title{
On Automatic-Verification Pattern Generation for SoC With Port-Order Fault Model
}

\author{
Chun-Yao Wang, Student Member, IEEE, Shing-Wu Tung, Member, IEEE, and Jing-Yang Jou, Senior Member, IEEE
}

\begin{abstract}
Embedded cores are being increasingly used in the designs of large system-on-a-chip (SoC). Because of the high complexity of SoC, the design verification is a challenge for system integrators. To reduce the verification complexity, the port-order fault (POF) model has been used for verifying core-based designs (Tung and Jou, 1998) . In this paper, we present an automatic-verification pattern generation (AVPG) for SoC design verification based on the POF model and perform experiments on combinational and sequential benchmarks. Experimental results show that our AVPG can efficiently generate verification patterns with high POF coverage.
\end{abstract}

Index Terms-Automatic-verification pattern generation (AVPG), design verification, IEEE P1500, port-order fault (POF), SoC, undetected port sequence (UPS).

\section{INTRODUCTION}

$\mathbf{S}$ PURRED by process technology leading to the availability of more than one-million gates per chip and more stringent requirements upon time-to-market and performance constraints, system-level integration and platform-based design [2] are evolving as a new paradigm in system designs. A multitude of components that are needed to implement the required functionality make it hard for a company to design and manufacture an entire system in time and within reasonable cost. Hence, design reuse and reusable building blocks (cores) trading are becoming popular in the system-on-a-chip (SoC) era. However, present design methodologies are not enough to deal with cores which come from different design groups and are mixed and matched to create a new system design. In particular, verifying whether a design satisfies all requirements is one of the most difficult tasks.

Verification is a process used to demonstrate the functional correctness of a design. Testing is a process that verifies whether the design was manufactured correctly. Fig. 1 shows the reconvergent paths model for both verification and testing [3]. During testing, the finished silicon is reconciled with the netlist that was submitted for manufacturing. Therefore, when a design is claimed to be fully tested, i.e., $100 \%$ fault coverage, under a fault model, such as stuck-at fault (SAF) model, that means it is manufactured correctly. However, we still cannot guarantee that the chip satisfies the design specification if we do not verify it properly before manufacturing. The chip may be a manufac-

Manuscript received May 23, 2001; revised November 1, 2001. This work was supported in part by the R.O.C. National Science Council under Grant NSC89-2215-E-009-009. This paper was recommended by Associate Editor J. H. Kukula.

The authors are with the Department of Electronics Engineering, National Chiao Tung University, Hsinchu 300, Taiwan, R.O.C. (e-mail: wcyao@eda.ee.nctu.edu.tw).

Publisher Item Identifier S 0278-0070(02)02471-5.

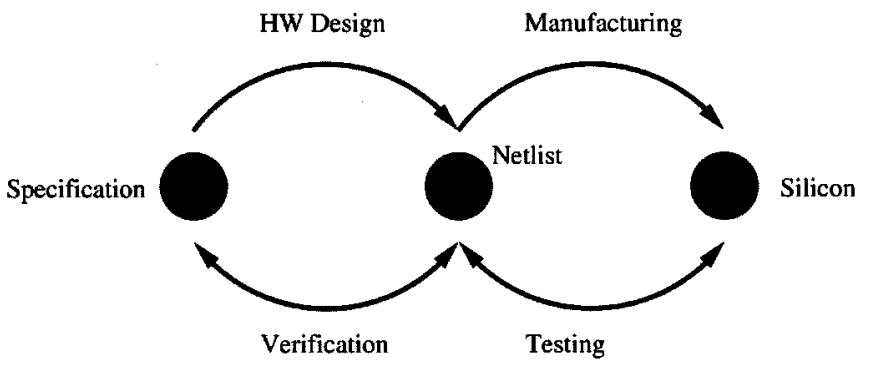

Fig. 1. Reconvergent paths model for both verification and testing.

tured correctly but designed incorrectly chip. Thus, designers spend about $70 \%$ of their efforts on the verification. But design verification is still on the critical path of the design flow [3].

Usage of cores divides the integrated circuit (IC) design community into two groups: core providers and system integrators. In traditional system-on-board ( $\mathrm{SoB}$ ) design, the components that go from providers to system integrators are ICs, which are designed, verified, manufactured, and tested. The system integrator verifies the design by using these components as fault-free building blocks. SoB verification is limited to detecting faults in the interconnection among the components. Similarly, in SoC design, the components are cores. The system integrator verifies the design by using the cores as design error-free building blocks. Based on this assumption, SoC verification could be focused on detecting the misplacements of the interconnection among the cores as the first step. This higher level of abstraction decreases the complexity of design verification on a system chip and reduces the time on design verification of the entire system.

Most previous work in testing interconnection focused on the development of deterministic tests for interconnection between chips at the board level [4], [5]. The extension of these board-level testing methods to core-based design verification is inappropriate. In the interconnection testing phase, only system-level interconnection is tested. The basic assumption for a system under test is based on the system design being correct and the faults are due to manufacturing defects on interconnection among components. For core-based $\mathrm{SoC}$ design verification, the system is not fully verified yet and the most of system design errors are due to incorrect designs of interconnection among predesigned cores. The methods proposed to test interconnection do not guarantee that the interconnection among cores is correct.

The focus of core-based design verification should be on how the cores communicate with each other [6]. By creating the testbenches at a higher level, a connectivity-based design fault model, port-order fault (POF), proposed in [7] is used for reducing the time on core-based design verification [1]. In [1], 


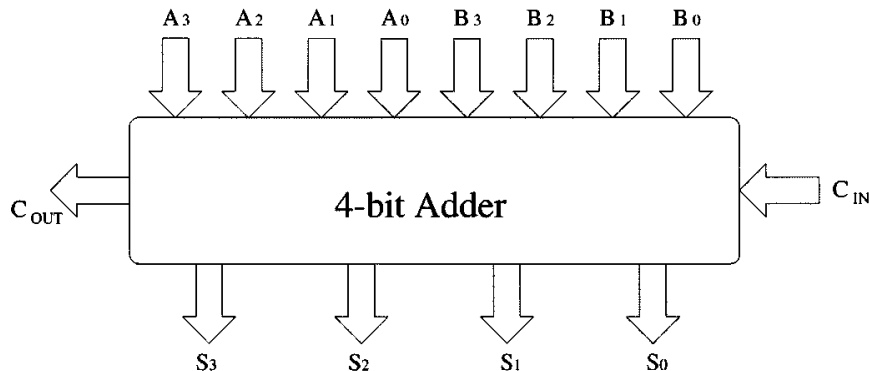

Fig. 2. A fault-free 4-bit adder.

Tung et al. proposed a verification pattern generation algorithm based on the POF model. The algorithm cooperates with the SAF automatic test pattern generation to generate the verification pattern set for detecting the simple POF (SPOF) (two ports misplaced at a time). This approach needs the circuit netlist to generate the verification patterns, thus, it loses the capability of integrating different levels of embedded cores (soft cores, firm cores and hard cores) into a system. For example, this algorithm cannot generate verification patterns for soft cores. Furthermore, the simplified SPOF model is not enough to deal with all possible misplacements occurred in a real design during the cores integration phase. In this automatic-verification pattern generation (AVPG), however, all possible misplacements among the ports of cores are considered rather than two ports misplacements. Furthermore, it only uses the simulation information of the core rather than the circuit netlist to generate the verification pattern set. This loosened requirement allows the different levels of cores being integrated together for the POF verification.

The POF AVPG is integrated into the SIS [8] environment. Experiments are conducted on combinational and sequential benchmarks, such as ISCAS-85, ISCAS-89, and MCNC benchmarks. Experimental results show that the AVPG can efficiently generate verification pattern sets with high POF coverage in the proposed verification environment which exploits the IEEE P1500 Standard for embedded core test (SECT) [9].

The remainder of this paper is organized as follows. The POF model and some terminologies are introduced in Section II. Section III describes the mechanism of conducting POF verification. The POF AVPG is presented in Section IV. Section V presents experimental results. Section VI concludes the paper.

\section{PReliminary}

The POF model belongs to the group of pin-faults models [10], which assumes that a faulty cell has at least two input-output (I/O) ports misplaced. It also assumes that the components are fault free and only the interconnection among the components could be faulty. There are three types of POFs [7].

Definition 1: The type-I POF is at least one input $x_{k}$ misplaced with one output $y_{j}$, i.e., the input ports of faulty core $X^{\prime}=\left\{x_{1}, \ldots, x_{i}, \ldots, y_{j}, \ldots, x_{n} \mid 1 \leq\right.$ $i \leq n, i \neq k\}$ and output ports of faulty core $Y^{\prime}=$ $\left\{y_{1}, \ldots, y_{i}, \ldots, x_{k}, \ldots, y_{m} \mid 1 \leq i \leq m, i \neq j\right\}$.

Example 1: A fault-free 4-bit adder is shown in Fig. 2. The functionality of the adder is $\left\{C_{O U T}, S(3: 0)\right\}=A(3: 0)+$

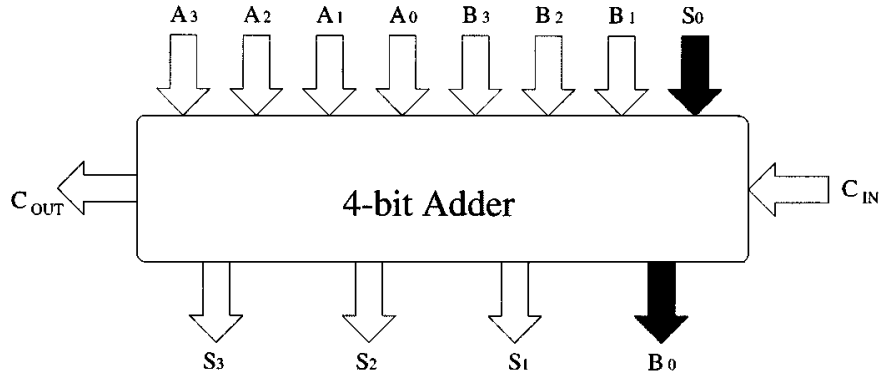

Fig. 3. A 4-bit adder with the type-I POF.

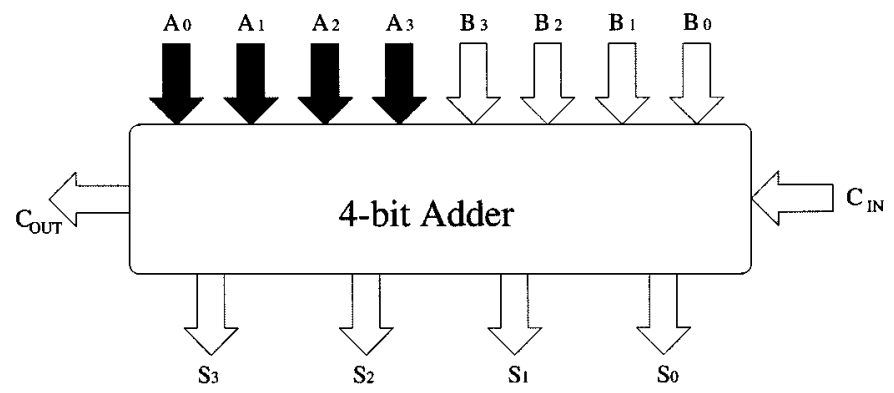

Fig. 4. A 4-bit adder with the type-II POF.

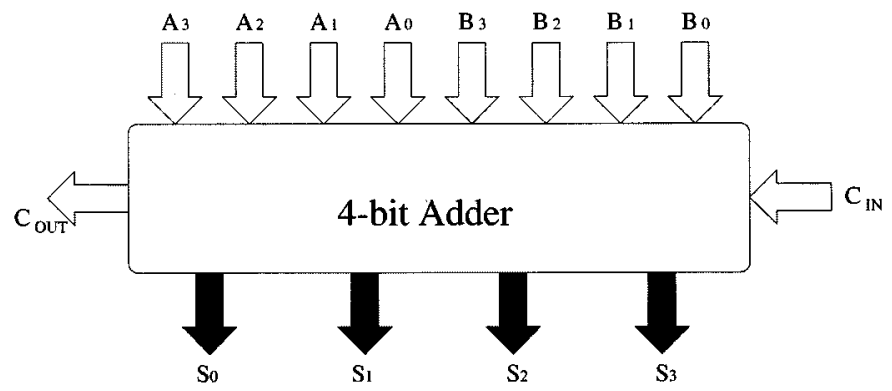

Fig. 5. A 4-bit adder with the type-III POF.

$B(3: 0)+C_{I N}$. An example of the type-I POF is shown in Fig. 3. Input port $B_{0}$ is misplaced with output port $S_{0}$.

Definition 2: The type-II POF is at least two input ports misplaced, i.e., the input ports of the fault-free core $X=\left\{x_{1}, \ldots, x_{i}, \ldots, x_{j}, \ldots, x_{n} \mid i \neq j\right\}$, but the input ports of the faulty core $X^{\prime}=\left\{x_{1}, \ldots, x_{j}, \ldots, x_{i}, \ldots, x_{n} \mid i \neq j\right\}$.

Example 2: An example of the type-II POF is shown in Fig. 4. Input ports $A(3: 0)$ are misplaced with input ports $A(0: 3)$. The order of the input ports $A(3: 0)$ is reversed.

Definition 3: The type-III POF is at least two output ports misplaced, i.e., the output ports of the fault-free cell $Y=\left\{y_{1}, \ldots, y_{i}, \ldots, y_{j}, \ldots, y_{m} \mid i \neq j\right\}$, but the output ports of the faulty cell $Y^{\prime}=\left\{y_{1}, \ldots, y_{j}, \ldots, y_{i}, \ldots, y_{m} \mid i \neq j\right\}$.

Example 3: An example of the type-III POF is shown in Fig. 5. Output ports $S(3: 0)$ are misplaced with output ports $S(0: 3)$. The order of the output ports $S(3: 0)$ is reversed.

Because the misplaced output port in the type-I POF has no driving source, it has invariant value (typical high impedance) that could be detected by applying any verification patterns. Therefore, the verification patterns for the type-II POF can also detect the type-I POF.

In Fig. 6, it shows a generic system chip which contains six cores, BLK1 $\sim$ BLK6. The verification on type-III POF of 


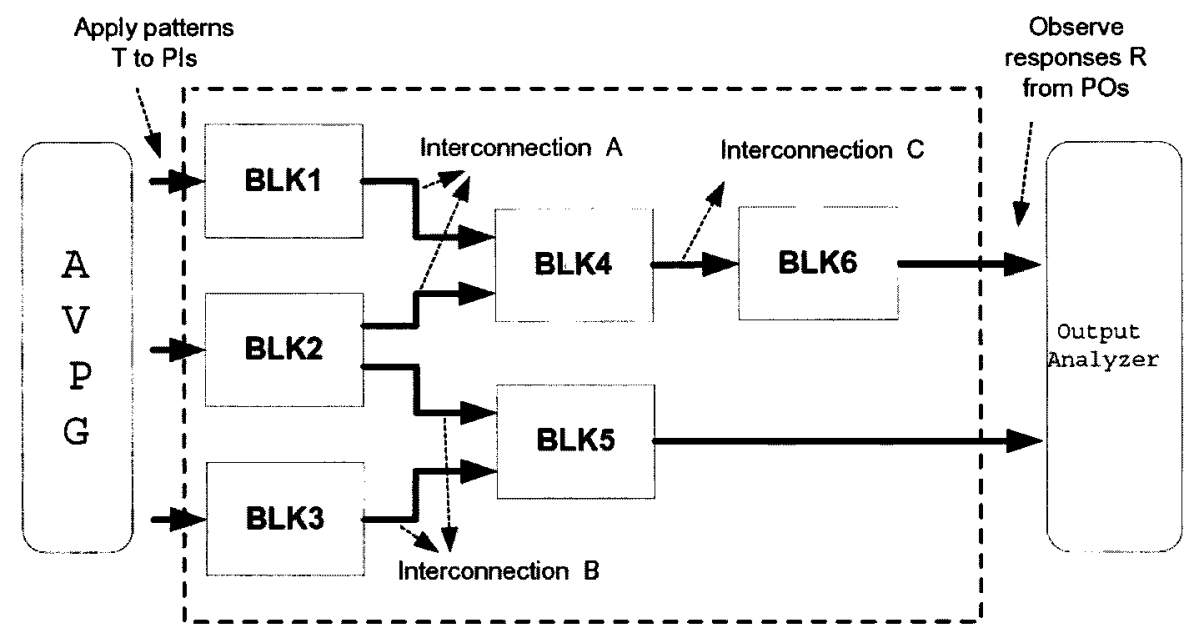

Fig. 6. Generic verification scheme.

$\mathrm{BLK} 1 \sim \mathrm{BLK} 4$ is equivalent to the verification on type-II POF of BLK4 BLK6. Therefore, if the type-II POF of all cores in the system are verified, the type-III POF are also verified simultaneously. Hence, in this paper, the AVPG generates the verification patterns for detecting the type-II POF solely.

Definition 4: A port sequence is an input port numbers permutation that indicates the relative positions among these input ports.

Definition 5: The fault-free port sequence is a port sequence that none of the input ports is misplaced. For an $N$-input core, the input ports are numbered from 1 to $N$. The number of the input port numbers permutation is $N$ ! and these $N$ ! permutations represent the $N$ ! port sequences of the core. Except the fault-free port sequence, the remaining $(N !-1)$ port sequences represent the core with some particular POFs and are called faulty port sequences. In this paper, the POFs and the faulty port sequences are used exchangeably.

\section{INTEGRATION VERIFICATION}

Using core-based design methodology could reduce the time-to-market for system chips. However, the verification efforts of system chip are still proportional to the design complexity. Both simulation and verification technique cannot reduce the total verification time effectively if those predesigned and preverified blocks are to be verified exhaustively during the integration phase. Therefore, in this section, we introduce the IEEE P1500, which is a standard under development and is used for embedded core testing, to reduce the complexity of design verification. Besides, we will explain how to use the POF verification pattern set for verifying integrated SoC designs.

Fig. 6 depicts a generic verification scheme for the core-based system chip. Since these cores, BLK1 BLK6, are preverified, the verification efforts during the integration phase should focus on the interconnection among the cores. To verify the interconnection among the BLK1 BLK6, designers apply the patterns $T$ to primary inputs (PIs) of the integrated design, then compare the responses $R$ to the expected results in primary outputs (POs). If the responses $R$ are inconsistent with the expected ones, some interconnection are misplaced. The generation of the patterns $T$

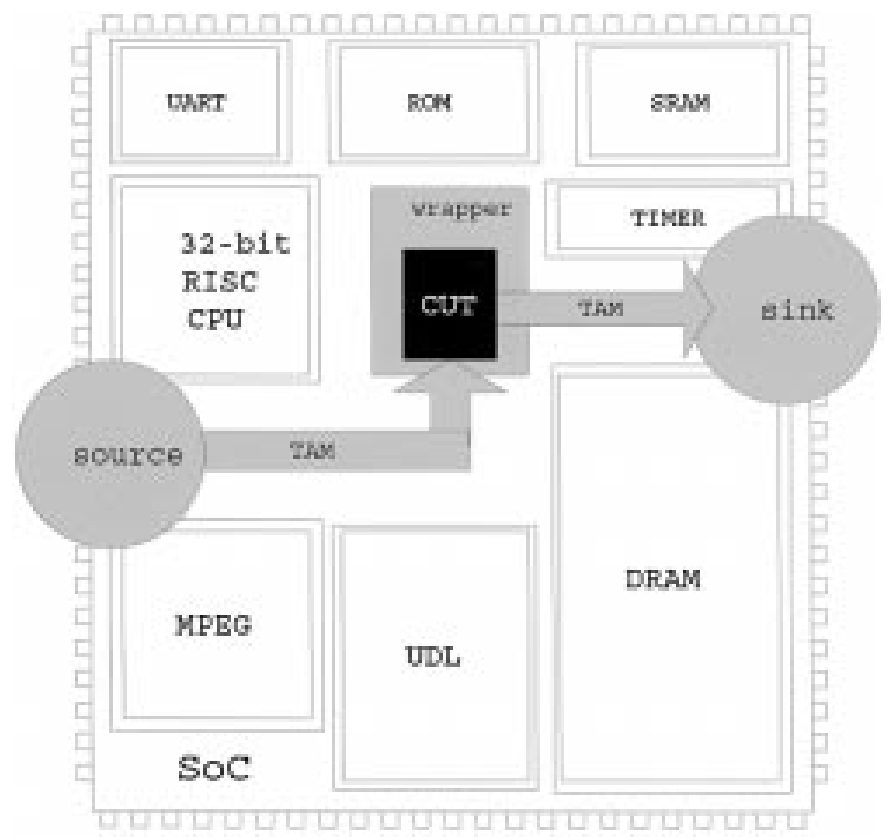

Fig. 7. Generic test access architecture for embedded cores.

depends on the functionalities of BLK1 BLK6. As the complexity of cores increase or more cores are involved in the SoC integration, the patterns $T$ become harder to generate.

To conquer this problem, we exploit the technique of design for testability to conduct verification. The solution is the IEEE P1500 SECT [9], [11]. The IEEE P1500 SECT is a standard under development that aims at improving ease of reuse and facilitating interoperability with respect to the test of core-based chips. The IEEE P1500 working group has suggested a modulelevel boundary-scan structure which is very similar to the IEEE 1149.1 (JTAG) [12] structure. The structure, called "wrapper", is a thin shell around the core that allows intercore and intracore test functions to be carried out via a test access mechanism (TAM). The TAM itself is user defined and is not specified in the draft standard [13]. Fig. 7 depicts a generic access architecture for testing embedded core schematically [11]. Fig. 8 shows a typical configuration of how the chip-level connection of the cores might be connected in one serial TAM and one parallel 


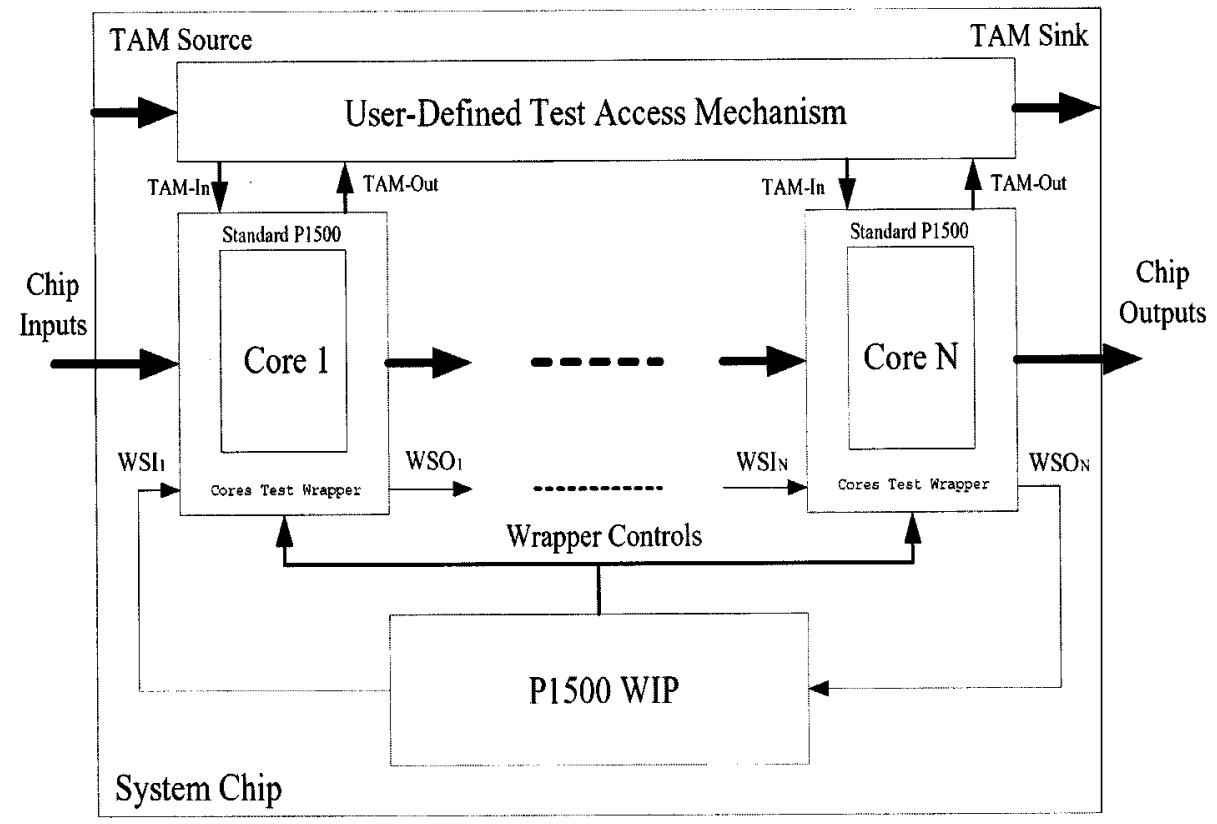

Fig. 8. A typical configuration of the cores integration using the IEEE P1500 architecture.

TAM per core [14]. The IEEE P1500 establishes the mechanism that the test patterns of any circuits under test given by core providers can be applied to PIs of the system chip (source) and propagated to POs of the system chip (sink) via user-defined TAMs.

Most of the POFs occur because of human introduced errors in the SoC design process. These human errors are normally introduced by misinterpretation of the design specification of interconnection. Usually, the IP cores are integrated by system integrators and the test structure among the cores is constructed by test engineers. That is to say, the cores which are connected for normal operation is done by an individual in the core integration phase and the cores which are connected for test mode is done by another individual in the test insertion phase. These two mode connections are independent. Therefore, the likelihood of making the same mistake is very small.

A straightforward core integration methodology is used and the system is integrated blockwise. As a block is added into the system, the verification patterns for the added block are generated and applied to the integrated system for the interconnection verification.

We exploit the IEEE P1500 wrappers and user defined TAMs to propagate the verification patterns from PIs to the wrappers in the predecessor of the core under verification (CUV) and to propagate responses of the CUV to POs. The IEEE P1500 wrapper was proposed with a few predefined operations, such as core-internal test, core-external test, bypass, isolation, and normal modes.

In order to verify the interconnection among the CUV and its predecessors, the CUV is set in normal mode which allows the CUV to function in its normal system operation. The predecessors connected to the CUV directly are set in external test mode which allow verifying the interconnected wiring between cores via the ordinary I/O ports in the core wrappers. The other prede- cessors of the CUV are all set in bypass mode which allow the stimuli being bypassed through cores to the CUV.

For example, assume the BLK1 BLK6 have to be integrated into a system as shown in Fig. 6. In the beginning, the BLK1 BLK3 are added into the system. Since these blocks do not have any predecessors, it is not necessary to conduct the POF verification. As the BLK4 is added into the system, the BLK1 and BLK2 are the predecessors that are directly connected to it. In order to verify the interconnection A among these blocks, the BLK4 is set in normal mode and the BLK1 and BLK2 are set in external test mode to propagate the POF stimuli from PIs through the wrappers (of BLK1 and BLK2) to the inputs of the BLK4 as shown in Fig. 9. Hence, the verification patterns can easily go through the system from PIs to POs and verify the interconnection A. If there are any misplacements in the interconnection A, the inconsistent results will be observed in the output analyzer. Similarly, as the BLK5 is added into the system, it is set in normal mode. The BLK2 and BLK3 are set in external test mode as shown in Fig. 10. And so forth, as the BLK6 is added into the system, it is set in normal mode. The BLK4 is the predecessor that is directly connected to the BLK6. Hence, it is set in external test mode. The BLK1 and BLK2 are the other predecessors of the BLK6, they are set in bypass mode. This is shown in Fig. 11.

This verification mechanism allows us solely focusing on the functionality of the added block when generating the verification pattern set and reduce the complexity of POF verification. Note that for verifying the interconnection of an added core, the core is exercised via the normal operation path. This is because system integrators possibly have misunderstanding about the correct interconnection among cores. Only the consistency of simulation results and expected results can guarantee the correctness of integration.

By using the IEEE P1500 test structure for the POF verification, we do not introduce any more hardware overhead in the 


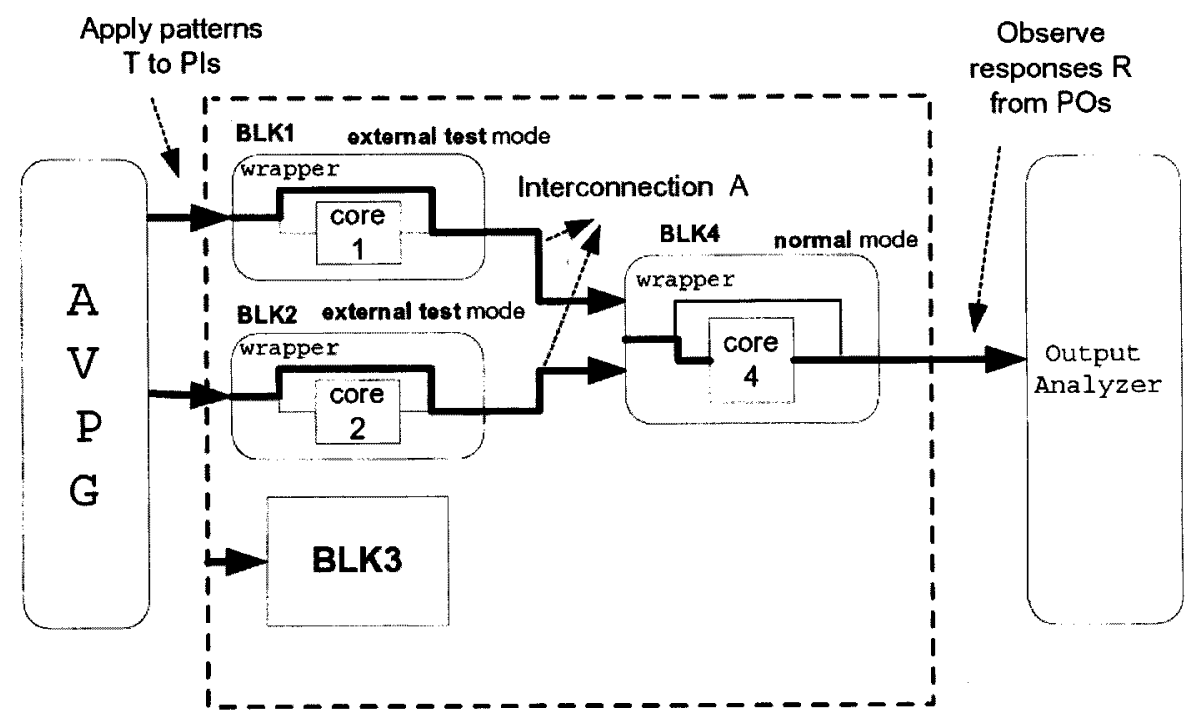

Fig. 9. The POF verification when integrating the BLK4.

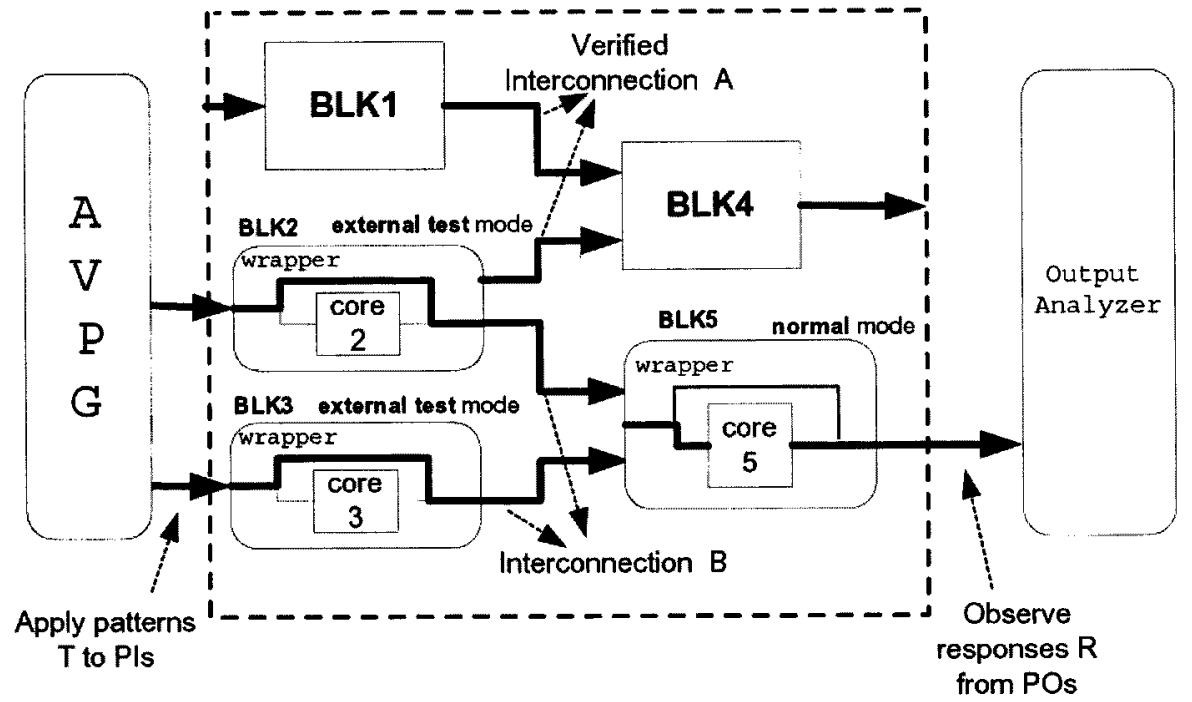

Fig. 10. The POF verification when integrating the BLK5.

chip implementation. In fact, we reuse the hardware overhead incurred in the testing phase.

\section{THE POF-BASED AVPG}

This section describes the AVPG that is shown in the left part of Fig. 11.

\section{A. The Combinational AVPG}

1) UPS Representation: Typically, the automatic pattern generator for functional errors, such as transition fault [15] or manufacturing faults, such as SAF [16], builds fault list explicitly first to explore how many faults have to be detected with specific patterns, then generates random patterns and deterministic patterns. For the POF-based AVPG, however, the fault list cannot be enumerated explicitly. This is because the total number of POFs in an $N$-input core is $N !-1$. This number grows rapidly when $N$ increases, for instance, as $N=20$, $N !-1 \approx 2.4 \times 10^{18}$, as $N=69, N !-1 \approx 1.7 \times 10^{98}$. Instead, an implicit representation is used to indicate the remaining undetected port sequences (UPSs) during the verification pattern generation. When the remaining UPS becomes empty, all POFs are detected. In the pattern generation stage, the heuristic patterns are generated instead of random patterns and deterministic patterns. In other words, the approach is to "search" proper patterns for the POF verification in a systematic way.

In the beginning, Example 4 demonstrates the implicit UPSs representation.

Example 4: Given an 8-input core, the input ports are numbered from 1 to 8 . The UPS's representation (12345678) represents the UPSs that caused by all possible misplacements among the port numbers in the same group, i.e., Port 1 to Port 8 . The number of undetected POFs is $8 !-1$ and the one in the $8 !-1$ accounts for the fault-free port sequence. The UPS's representation (125)(4)(3678) indicates the UPSs that caused by all possible misplacements among the port numbers 1,2, and 5 and/or all possible misplacements among the port numbers $3,6,7$, and 8 . The number of the undetected 


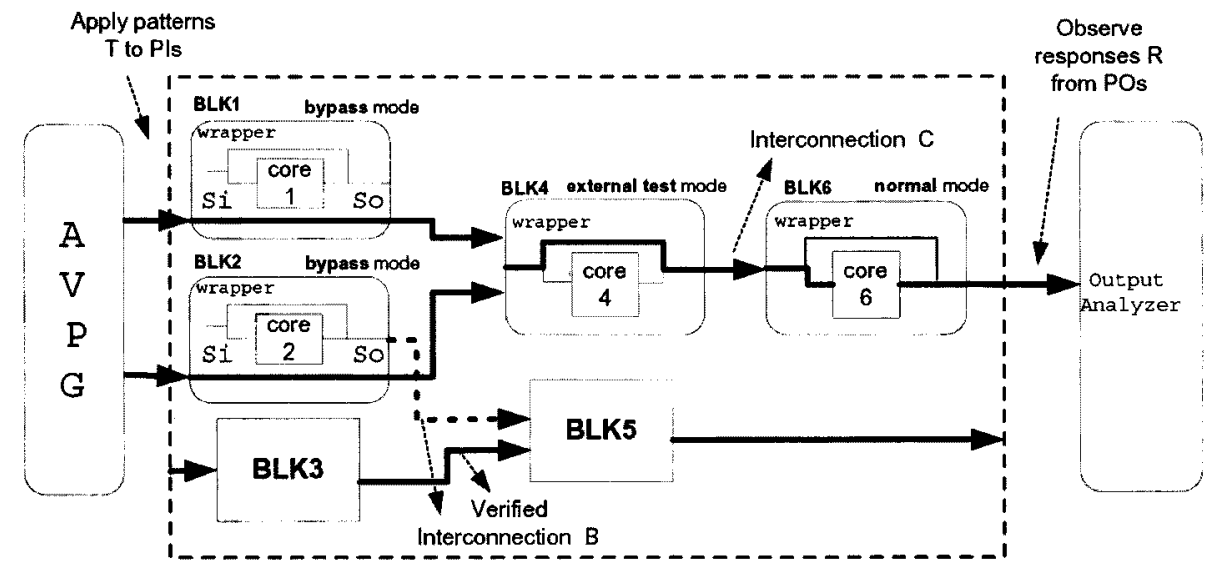

Fig. 11. The POF verification when integrating the BLK6.

POFs is $3 ! \times 1 ! \times 4 !-1$. Note that the port number 4 is the only one element in the second group. It means that the port sequences whose port number 4 in the wrong position are not represented by this UPS's representation. The order of the groups in the UPS's representation is irrelevant, neither is the order of the numbers in each UPS's group. For example, the UPSs (125)(4)(3678) can also be expressed as (4)(215)(8763). The UPSs (12)(3)(4)(5)(6)(78) contain four port sequences and they are 12345678, 21345678, 12345687 , and 21345687. However, the UPS's representation of the port sequences 21345678 and 12345687 is also (12)(3)(4)(5)(6)(78). This is because we always use one UPS's representation to express all UPSs. The UPS representation (1)(2)(3)(4)(5)(6)(7)(8) has eight groups and each group has only one element, therefore, no misplacement could be occurred in each group. The number of the undetected POFs is $1 ! \times 1 ! \times 1 ! \times 1 ! \times 1 ! \times 1 ! \times 1 ! \times 1 !-1=0$. Hence, (1)(2)(3)(4)(5)(6)(7)(8) represents $8 !-1$ POFs are all detected. If the UPS's representation is induced from (12345 678) to (1)(2)(3)(4)(5)(6)(7)(8), all POFs are detected.

2) The Verification Pattern Generation: This section describes the verification pattern generation algorithm, which is the foundation of our AVPG.

Definition 6: For an $N$-input combinational core, the exhaustive pattern set is defined as $\Phi^{N}$. The size of $\Phi^{N}$ is the number of patterns in $\Phi^{N}$ and is denoted as $\left|\Phi^{N}\right|$ and $\left|\Phi^{N}\right|$ equals $2^{N}$.

Definition 7: The set that consists of all patterns with $m$ ones and $(N-m)$ zeroes is denoted as $\Theta_{m}^{N}$, where $m \in[0,1,2, \ldots, N-1, N]$. The size of $\Theta_{m}^{N}$ is the number of patterns in $\Theta_{m}^{N}$ and is denoted as $\left|\Theta_{m}^{N}\right|$ and $\left|\Theta_{m}^{N}\right|$ equals $\left(\begin{array}{c}N \\ m\end{array}\right)$ where $\left(\begin{array}{l}N \\ m\end{array}\right)=N ! /(m !(N-m) !)$.

Example 5: For a 4-input core, $\Phi^{4}$ is the exhaustive pattern set with 4 bits. $\left|\Phi^{4}\right|=2^{4}=16 . \Theta_{0}^{4}=\{0000\},\left|\Theta_{0}^{4}\right|=\left(\begin{array}{l}4 \\ 0\end{array}\right)=$ 1. $\Theta_{1}^{4}=\{1000,0100,0010,0001\},\left|\Theta_{1}^{4}\right|=\left(\begin{array}{l}4 \\ 1\end{array}\right)=4$. $\Theta_{2}^{4}=$ $\{1100,1010,1001,0110,0101,0011\},\left|\Theta_{2}^{4}\right|=\left(\begin{array}{l}4 \\ 2\end{array}\right)=6, \Theta_{3}^{4}=$ $\{1110,1101,1011,0111\},\left|\Theta_{3}^{4}\right|=\left(\begin{array}{l}4 \\ 3\end{array}\right)=4 . \Theta_{4}^{4}=\{1111\}$, and $\left|\Theta_{4}^{4}\right|=\left(\begin{array}{l}4 \\ 4\end{array}\right)=1$.

For an $N$-input core, $\Phi^{N}$ can be used for verifying the functionality of the core completely. However, $\left|\Phi^{N}\right|$ equals $2^{N}$ and this number grows rapidly when $N$ increases. Hence, functional verification using $\Phi^{N}$ as verification pattern set is impractical. We have to use another strategy to generate proper patterns for verification.
The following paragraphs are going to introduce the three steps in the pattern generation algorithm: fault activation, fault propagation, and fault domination. They are operated in sequence and iteratively and are described in detail in the subsequent sections.

a) Fault Activation: Fault activation is the most important procedure in the algorithm. If the fault effect is not activated, it surely cannot be propagated out. To activate a POF, the logic assignments of the corresponding input ports cannot be all the same. For example, to activate the faulty port sequence 1243, the assignments of Port 3 and Port 4 have to be different, either Port 3 is assigned zero, Port 4 is assigned one or vice versa. All $N$ ! -1 POFs have to be activated during the verification pattern generation. The following theorem states the completeness of the POF activation.

Theorem 1: $\Theta_{m}^{N}$ where $m \in[1,2, \ldots, N-2, N-1]$ can activate all $(N !-1)$ POFs.

Proof: Given a pattern $T \in \Theta_{m}^{N}$, there are $m$ ones and $(N-m)$ zeroes in $T$. After any port misplacements, the pattern $T$ turns to $T^{\prime}$ where $T^{\prime}$ must also have $m$ ones and $(N-m)$ zeroes $\left(T^{\prime} \in \Theta_{m}^{N}\right)$. Because $\Theta_{m}^{N}$ contains all patterns with $m$ ones and $(N-m)$ zeroes, for each POF, there must exist a pair of patterns $\left(T, T^{\prime}\right) \in \Theta_{m}^{N}$ that corresponds to the original pattern $T$ and the activated pattern $T^{\prime}$. Thus, $(N !-1)$ POFs are all activated by $\Theta_{m}^{N}$ for $m \in[1,2, \ldots, N-2, N-1]$. Q.E.D.

Example 6: Fig. 12 shows an example to illustrate that $\Theta_{2}^{4}$ can activate all (4! - 1) POFs. In Fig. 12(a), the pattern $T, 1100$, is the original pattern. After the misplacement of Port 2 and Port 3 , the real pattern applied into the core is $T^{\prime}, 1010$. The pattern $T$ is different with $T^{\prime}$, hence, the faulty port sequence 1324 is activated. In Fig. 12(b), the patterns in the column $T$ represent the original patterns and the column $T^{\prime}$ represents the real patterns applied into the core after the port misplacements shown in the second column. In the second row, the pattern pair (1100, 1010) can activate faulty port sequences $1324,1423,2314$, and 2413. In the third row, the pattern pair $(1100,1001)$ can activate faulty port sequences $1342,1432,2341$, and 2431 and so on. $(4 !-1)$ POFs are all activated by pattern pairs $\left(T, T^{\prime}\right) \in \Theta_{2}^{4}$. To activate a faulty port sequence, the pattern pair $\left(T, T^{\prime}\right)$ is not unique. For example, to activate the faulty port sequence 1243, the only requirement is that the assignments of Port 3 and Port 4 have to be different, therefore, both pattern pair $(1010,1001)$ 


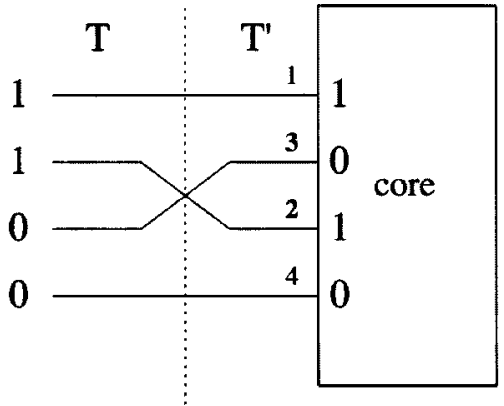

activated faulty

port sequence 1324

(a)

\begin{tabular}{|c|c|c|}
\hline $\mathrm{T}$ & $\begin{array}{l}\text { activated faulty } \\
\text { port sequences }\end{array}$ & $\mathrm{T}^{\prime}$ \\
\hline 1100 & $\begin{array}{l}1324,1423 \\
2314,2413\end{array}$ & 1010 \\
\hline 1100 & $\begin{array}{l}1342,1432 \\
2341,2431\end{array}$ & 1001 \\
\hline 1100 & $\begin{array}{l}3124,4123 \\
3214,4213\end{array}$ & 0110 \\
\hline 1100 & $\begin{array}{l}3142,4132 \\
3241,4231\end{array}$ & 0101 \\
\hline 1100 & $\begin{array}{l}3412,4312 \\
3421,4321\end{array}$ & 0011 \\
\hline 1010 & 2134 & 0110 \\
\hline 1010 & 2143 & 0101 \\
\hline $\begin{array}{r}1010 \\
(0110)\end{array}$ & 1243 & $\begin{array}{c}1001 \\
(0101)\end{array}$ \\
\hline
\end{tabular}

(b)

Fig. 12. The $4 !-1$ POFs are all activated by patterns in $\Theta_{2}^{4}$.

and $(0110,0101)$ can activate this faulty port sequence, that is shown in the last row of Fig. 12(b).

Corollary 1: $\Theta_{0}^{N}$ and $\Theta_{N}^{N}$ cannot be the verification patterns.

According to Theorem 1 and Corollary 1, we can arbitrary apply $\Theta_{m}^{N}$ for $m \in[1,2, \ldots, N-2, N-1]$ to the inputs of the core to activate $(N !-1)$ POFs. However, note that

$$
\begin{aligned}
& \left.\left|\Theta_{m}^{N}\right| \leq\left|\Theta_{m+1}^{N}\right|, \quad \text { for } m \in\left[1,2, \ldots, \mid \frac{(N-1)}{2}\right]\right] \\
& \left|\Theta_{m}^{N}\right| \leq\left|\Theta_{m-1}^{N}\right|, \quad \text { for } m \in\left[\left[\frac{(N+1)}{2} \mid, \ldots, N-1\right] .\right.
\end{aligned}
$$

Equations (1) and (2) show that the $\left|\Theta_{m}^{N}\right|$ is smaller when $m$ is closer to the end points of interval $[1,2, \ldots, N-1]$. Therefore, to minimize the number of simulation patterns, we select $m$ from one up to $\lfloor N / 2\rfloor$ or from $N-1$ down to $\lfloor N / 2\rfloor$. b) Fault Propagation: The simulation results of the applied patterns are observed to determine which activated POFs are propagated to POs. The fault effects are propagated to POs if there exists different responses among these input patterns.

c) Fault Domination: If a POF caused by the misplacement of input ports $x_{i}$ and $x_{j}$ and denoted as $\operatorname{POF}\left(x_{i}, y_{j}\right)$ is detected by a pattern $T$, we can figure out what the other POFs are detected by $T$ simultaneously. This characteristic of a POF pattern can reduce the size of the verification pattern set and is stated in Theorem 2. At this stage, the remaining UPSs are also calculated so that more verification patterns can be generated accordingly.

Theorem 2: For an $N$-input core, assume the $\operatorname{POF}\left(x_{i}, x_{j}\right)$ can be detected by a verification pattern $T \in \Theta_{m}^{N}$, then $T$ can actually detect $m ! \times(N-m) ! P O F$ s in total. This characteristic is called the domination property of a POF pattern.

Proof: Suppose the output of the verification pattern $T$ is $A$. Because $T$ detect the $\operatorname{POF}\left(x_{i}, x_{j}\right)$, the logic value assignments of $x_{i}$ and $x_{j}$ in $T$ must be different $\left(x_{i}=0, x_{j}=1\right.$ or vice versa). The exchange of $x_{i}$ and $x_{j}$ reform a new pattern $T^{\prime}$ and the output of $T^{\prime}$ is $B$ where $B \neq A$. This is because $T$ is a verification pattern for the $\operatorname{POF}\left(x_{i}, x_{j}\right)$, the outputs of $T$ and $T^{\prime}$ must be different. If the zeroes in $T^{\prime}$ are misplaced with themselves, or the ones in $T^{\prime}$ are misplaced with themselves, the representation of the pattern is still $T^{\prime}$ and the output remains $B$. These additional misplacements combined with the $\operatorname{POF}\left(x_{i}, x_{j}\right)$ are all detected by $T$ (output value $B \neq A$ ) and the total number of these POFs are $m ! \times(N-m) !$. Therefore, the verification pattern $T$ can actually detect $m ! \times(N-m)$ ! POFs. Q.E.D.

Example 7: For a 5-input core, assume a verification pattern 11000 detects the $\operatorname{POF}(2,3)$ and the output of the pattern 11000 is $A$. Because the verification pattern 11000 detects the $\operatorname{POF}(2,3)$, the output of the verification pattern 10100 must not be $A$ (assume it is $B$ ). The additional misplacements among the zeroes or between the ones in 10100 make the pattern 10100 intact and the output is still $B$. Therefore, these additional misplacements combined with $\operatorname{POF}(2,3)$ are all detected by 11000 and the amount of them are $2 ! \times 3 !$.

By using the property of the POF activation and domination and the implicit UPS's representation to handle the POF fault list, the process of the POF-based combinational AVPG algorithm is proposed.

The best way to demonstrate the algorithm is to discuss it with an example. Given an 8-input core, according to the UPS's representation, the initial UPSs are (12345 678). The number of UPSs is $8 !-1=40320-1$. The simulation results of $\Theta_{1}^{8}$ are shown in Fig. 13 and are represented in symbolic output representation. The simulation results depend on the functionality of the core. For the first pattern, 10000000, the first bit is one and the other bits are zeroes, the simulation output of 10000000 is represented as $A 0$. For the second pattern, 01000000 , the output is $B 0(B 0 \neq A 0)$ and so on. If the first pattern 10000000 is applied into the core and assume the interconnection is fault free, the port sequence is 12345678 and the output is $A 0$ as shown in Fig. 14(a). However, if the Port 1 and Port 2 are misplaced with each other, the port sequence becomes 21345678 . When the same pattern 10000000 is applied, the real pattern assigned into the core is 01000000 and the output becomes 


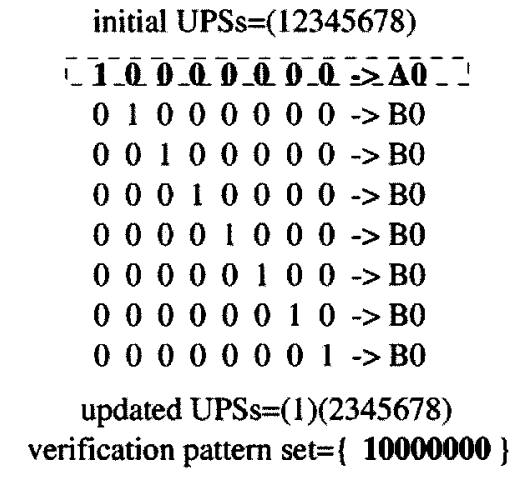

Fig. 13. The simulation results of $\Theta_{1}^{8}$ patterns.

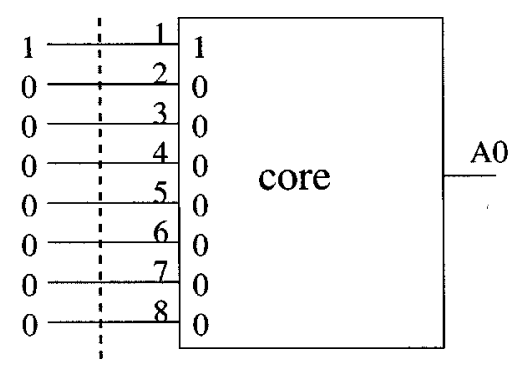

(a)

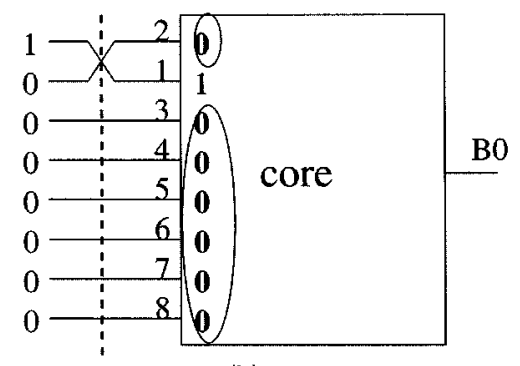

(b)

Fig. 14. Different outputs caused by the misplacement of Port 1 and Port 2.

$B 0$ as shown in Fig. 14(b). Because the fault-free output $A 0$ and faulty output $B 0$ are different, the faulty port sequence 21345678 can be detected by the pattern 10000000 . Furthermore, according to Theorem 2 , the Port $2 \sim 8$ are all zeroes, arbitrary port misplacements occurred among the ports $2 \sim 8$ after the $\operatorname{POF}(1,2)$ will evaluate to the same output $(B 0)$ and all of them are detected by the pattern 10000000 , too. This is shown in Fig. 14(b). Thus, the pattern 10000000 can detect $\{\mathrm{x} 1 \mathrm{xxxxxx}\}$ faulty port sequences where $x$ means any other port numbers. For another situation, if the Port 1 and Port 3 are misplaced with each other, the port sequence becomes 32145678 . When the same pattern 10000000 is applied, the real pattern assigned into the core is 00100000 and the output becomes $B 0$ again according to the simulation outputs of $\Theta_{1}^{8}$ shown in Fig. 13. Thus, 10000000 can detect this port sequence 32145678 and dominate port sequences $\{\mathrm{xx} 1 \mathrm{xxxxx}\}$. For the other ports, such as ports $4-8$, when Port 1 is misplaced with them, the results are similar. Thus, the faulty port sequences that are detected by 10000000 are $\{x 1 \mathrm{xxxxxx}, \mathrm{xx} 1 \mathrm{xxxxx}, \mathrm{xxx} 1 \mathrm{xxxx}, \mathrm{xxxx} 1 \mathrm{xxx}$,

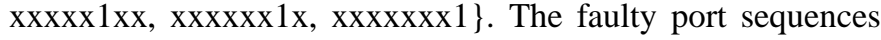
that are detected by 10000000 have been figured out, therefore, the faulty port sequences that cannot be detected by 10000000 are decided as well. These UPSs are $\{1 \mathrm{xxxxxxx}\}$ which can be represented as (1)(2345678) after the pattern 10000000 is added into the verification pattern set. These results are shown in Fig. 13.

When the second pattern 01000000 in Fig. 13 is applied into the core, since the first pattern 10000000 in Fig. 13 is the only one pattern which has different output with that of the second pattern, the second pattern only detects the $\operatorname{POF}(1,2)$ and dominates the port sequences $\{2 \operatorname{xxxxxxx}\}$. However, the port sequences $\{2 \mathrm{xx} x \mathrm{xxxx}\}$ have been detected by verification pattern 10000000 in the previous discussion (the updated UPSs are (1)(2 345678$)$. The second pattern does not detect any new faulty port sequences, therefore, it is not chosen as the verification pattern. Similarly, for the remaining patterns in $\Theta_{1}^{8}$, they have no contribution in reducing the size of UPSs, either. Thus, 10000000 is the only verification pattern added into the verification pattern set in this iteration.

In $\Theta_{1}^{8}$, the $i$ th bit is different with the other bits on the $i$ th pattern. We use the port number $i$ to represent the $i$ th pattern and group the port number $i$ according to the pattern outputs. In this example, the output of the first pattern is different with that of the other patterns, therefore, the grouping result of these port numbers is (1)(2345 678). This grouping result is the same with the updated UPSs obtained from our detailed discussion above. Hence, in the following discussion with applying $\Theta_{1}^{N^{\prime}}$ and $\Theta_{N^{\prime}-1}^{N^{\prime}}$ (only one bit is different with the other bits) to the input ports, the updated UPSs can be determined directly from the input port number grouping according to the simulation outputs of these patterns.

Definition 8: A single-element group (SEG) is a group that contains only one port number in the UPS's representation. A multiple-element group (MEG) is a group that contains more than one port numbers in the UPS's representation.

The group (1) in the updated UPSs (1)(2 345 678) is an SEG and the group (2345 678) in the updated UPSs (1)(2 345678 ) is a MEG. The physical meaning of the SEG is that the remaining UPSs are all irrelevant to the port in the SEG and the further pattern generation does not have to activate any POFs related to the port in the SEG. Therefore, when we search $\Theta_{7}^{8}$ for additional verification patterns, we find that the pattern 01111111 cannot activate any remaining POFs in the updated UPSs (1)(2 345 678). This is because the logic assignments in the ports $2 \sim 8$ of pattern 01111111 are all the same. Therefore, we exclude it from $\Theta_{7}^{8}$ to minimize the number of simulation patterns. The other patterns in $\Theta_{7}^{8}$ and their simulation outputs are shown in Fig. 15. We put an $X$ in the output of the pattern 01111111 to indicate the exclusion of this pattern from $\Theta_{7}^{8}$ simulations. The remaining patterns in $\Theta_{7}^{8}$ are grouped into three groups $\{10111111\},\{11011111\}$, and $\{11101111,11110111,11111011,11111101,11111110\}$ according to their outputs and the corresponding input port numbers grouping is (2)(3)(45678). These groups are sorted by size in ascending order. To add additional patterns into the verification pattern set, we always choose the group with the smallest size if it indeed can detect new faulty port sequences. In this example, when the first group $\{10111111\}$ is added into the verification pattern set, the updated UPSs become 
UPSs $=(1)(2345678)$

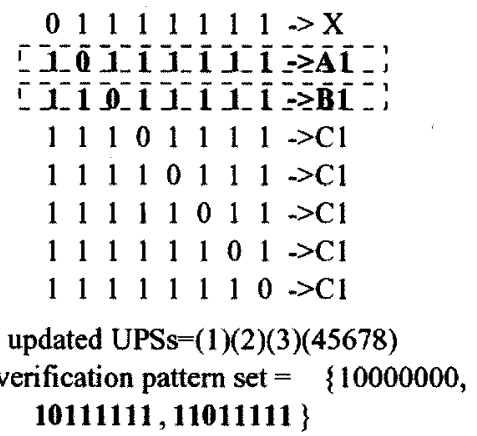

Fig. 15. The simulation results of $\Theta_{7}^{8}$ patterns.

$(1)(2)(3)(345678)$. When the second group $\{11011111\}$ is included into the verification pattern set, the updated UPSs become (1)(2)(3)(45678). These results come from the input port numbers grouping discussed above directly. For the third group, it has no contribution in reducing the size of UPSs further. Hence, it is not added into the verification pattern set. Consequently, in this iteration, the pattern $\{10111111\}$ and $\{11011111\}$ are added into the verification pattern set and the updated UPSs become (1)(2)(3)(45678). The size of the UPSs currently is reduced to $1 ! \times 1 ! \times 1 ! \times 5 !-1=120-1$.

Hence, the search for the verification pattern of the UPSs (1)(2)(3)(45678) is continued. The UPSs have four groups and are numbered from $G 1$ to $G 4$, i.e., $G 1$ is (1), $G 2$ is (2), $G 3$ is (3), and $G 4$ is (45678). $G 1 \sim G 3$ are SEGs and $G 4$ is a MEG. Note that if the UPSs (1)(2)(3)(45678) can be reduced to $(1)(2)(3)(4)(5)(6)(7)(8)$, the remaining POFs are all detected. The remaining POFs are only related to the ports in the MEGs and the further pattern generation is focused on the activation of these undetected POFs in the MEGs solely.

Then $\Theta_{2}^{8}$ are applied into the core. The patterns in $\Theta_{2}^{8}$ have two ones and six zeroes. These two ones in each pattern can be placed in the SEGs, MEGs or both. The SEG groups and the MEG group are placed into two sides, respectively, and all combinations of $\Theta_{2}^{8}$ patterns are listed in Fig. 16. In Fig. 16, the pattern set P1 does not activate any undetected POFs. Therefore, we have no need to simulate these patterns. In the pattern sets $\mathrm{P} 2$, $\mathrm{P} 3$, and $\mathrm{P} 4$, the MEG side assignments are $\Theta_{1}^{5}$ and all remaining POFs are activated according to Theorem 1 . For the pattern set P5, the MEG side assignments are $\Theta_{2}^{5}$ and also activate all remaining POFs. The SEG side assignments in each pattern set influence on the propagation of the activated fault effects. The propagation of fault effects is determined by the simulation outputs of these pattern sets. When the outputs of a pattern set are different, the fault effects are propagated out and the remaining UPSs could be further reduced.

In the pattern set P2, P3, since the outputs of the patterns in each set are all the same, therefore, the activated POFs cannot be propagated to POs and the UPSs remain (1)(2)(3)(45678). In the pattern set $\mathrm{P} 4$, the MEG assignments are $\Theta_{1}^{5}$ and the outputs are grouped into two groups. According to the previous discussion, the remaining UPSs can be determined directly by the input port numbers grouping. The grouping result is (45)(678), thus, the updated UPSs become $(1)(2)(3)(45)(678)$ when the patterns

\begin{tabular}{|c|c|c|c|c|}
\hline \multicolumn{5}{|c|}{ UPSs $=(1)(2)(3)(45678)$} \\
\hline \multirow{2}{*}{$\begin{array}{c}\text { pattern } \\
\text { set }\end{array}$} & \multirow{2}{*}{$\begin{array}{l}\text { SEG side } \\
(1)(2)(3) \\
\end{array}$} & MEG side & \multirow{2}{*}{ outputs } & \multirow{2}{*}{ description } \\
\hline & & $(45678)$ & & \\
\hline $\mathrm{P} 1$ & $\begin{array}{lll}1 & 1 & 0 \\
1 & 0 & 1 \\
0 & 1 & 1\end{array}$ & $\begin{array}{l}00000 \\
00000 \\
00000\end{array}$ & & $\begin{array}{l}\text { Two } 1 \text { s are placed } \\
\text { in the } S E G \text { side, } \\
\text { no POF activation. }\end{array}$ \\
\hline $\mathrm{P} 2$ & $\begin{array}{lll}1 & 0 & 0 \\
1 & 0 & 0 \\
1 & 0 & 0 \\
1 & 0 & 0 \\
1 & 0 & 0\end{array}$ & $\begin{array}{l}10000 \\
01000 \\
00100 \\
00010 \\
00001\end{array}$ & $\begin{array}{l}\mathrm{A} 2 \\
\mathrm{~A} 2 \\
\mathrm{~A} 2 \\
\mathrm{~A} 2 \\
\mathrm{~A} 2\end{array}$ & $\begin{array}{l}\text { One } 1 \text { is placed } \\
\text { in the SEG side, } \\
\text { and another } 1 \text { is } \\
\text { placed in the } \\
\text { MEG side. }\end{array}$ \\
\hline P3 & $\begin{array}{lll}0 & 1 & 0 \\
0 & 1 & 0 \\
0 & 1 & 0 \\
0 & 1 & 0 \\
0 & 1 & 0\end{array}$ & $\begin{array}{l}10000 \\
01000 \\
00100 \\
00010 \\
00001\end{array}$ & $\begin{array}{l}\text { A3 } \\
\text { A3 } \\
\text { A3 } \\
\text { A3 } \\
\text { A3 }\end{array}$ & $\begin{array}{l}\text { For the } \mathrm{P} 2 \text { and } \mathrm{P} 3 \text {, } \\
\text { fault effects are } \\
\text { not propagated } \\
\text { to POs. } \\
\text { For the P4, fault } \\
\text { effects are }\end{array}$ \\
\hline P4 & $\begin{array}{rrrr} & \mathbf{0} & \mathbf{0} & \mathbf{1} \\
1 & \mathbf{0} & \mathbf{n} & \mathbf{1} \\
0 & 0 & 1 \\
0 & 0 & 1 \\
0 & \mathbf{0} & 1\end{array}$ & $\begin{array}{c}\overline{\mathbf{1 0 0 0}} \overline{\mathbf{0}}- \\
-\mathbf{0 1 0 0 0} \\
00100 \\
00010 \\
00001\end{array}$ & $\begin{array}{c}-\overline{A 4}^{-1} \\
-\mathbf{A 4} \\
\text { B4 } \\
\text { B4 } \\
\text { B4 }\end{array}$ & $\begin{array}{l}\text { propagated to } \\
\text { POs. }\end{array}$ \\
\hline P5 & $\begin{array}{lll}0 & 0 & 0 \\
0 & 0 & 0 \\
0 & 0 & 0 \\
0 & 0 & 0 \\
0 & 0 & 0 \\
0 & 0 & 0 \\
0 & 0 & 0 \\
0 & 0 & 0 \\
0 & 0 & 0 \\
0 & 0 & 0\end{array}$ & $\begin{array}{l}11000 \\
10100 \\
10010 \\
10001 \\
01100 \\
01010 \\
01001 \\
00110 \\
00101 \\
00011\end{array}$ & $\begin{array}{l}\text { A5 } \\
\text { A5 } \\
\text { A5 } \\
\text { A5 } \\
\text { A5 } \\
\text { A5 } \\
\text { A5 } \\
\text { A5 } \\
\text { A5 } \\
\text { A5 }\end{array}$ & $\begin{array}{l}\text { Two 1s are placed } \\
\text { in the MEG side, } \\
\text { POFs are } \\
\text { activated but } \\
\text { not propagated. }\end{array}$ \\
\hline
\end{tabular}

00110000,00101000 ;

Fig. 16. The simulation results of $\Theta_{2}^{8}$ patterns.

$\{00110000,00101000\}$ are added into the verification pattern set. Because the outputs of patterns in the P5 are all the same, P5 is invalid for the POF verification.

In the next iteration, $\Theta_{6}^{8}$ are applied into the core and the simulation outputs are shown in Fig. 17. The only pattern set that activates and propagates the remaining POFs is P10. According to the outputs in the P10, the patterns are grouped into two sets $\mathrm{S} 1$ and $\mathrm{S} 2$ shown in Fig. 17. Since the assignments in the MEG side of P10 are not $\Theta_{1}^{N^{\prime}}$ and $\Theta_{N^{\prime}-1}^{N^{\prime}}$, the remaining UPS cannot be determined by input port numbers grouping directly. Here, we introduce the characteristic vector $(\mathrm{CV})$ grouping instead.

Definition 9: Given a set of patterns $S$, we count the number of digits 1 in the same bit position to form a vector with the same length. This vector is called the CV of $S$ and is denoted as $C V_{-} S$.

Definition 10: Given two pattern sets $S$ and $S^{\prime}$, if the patterns in the $S$ and $S^{\prime}$ are all identical, we said $S=S^{\prime}$, otherwise $S \neq$ $S^{\prime}$. If the corresponding bits in the $C V_{-} S$ and $C V_{-} S^{\prime}$ are all the same, we said $C V_{-} S=C V_{-} S^{\prime}$, otherwise $C V_{-} S \neq C V V_{-} S^{\prime}$.

The $C V V_{-} S 1[1: 8]$ is 22211022 and the $C V_{-S} S[1: 8]$ is 88855644 , as shown in Fig. 17. Since the updated UPSs are (1)(2)(3)(45)(678), the POFs related to ports in the SEG side are all detected, thus, we only consider the $C V_{-S} S 1[4: 8]$ and $C V_{-} S 2[4: 8]$ when analyzing the updated UPSs.

Lemma 1: One pattern set has only one CV. 


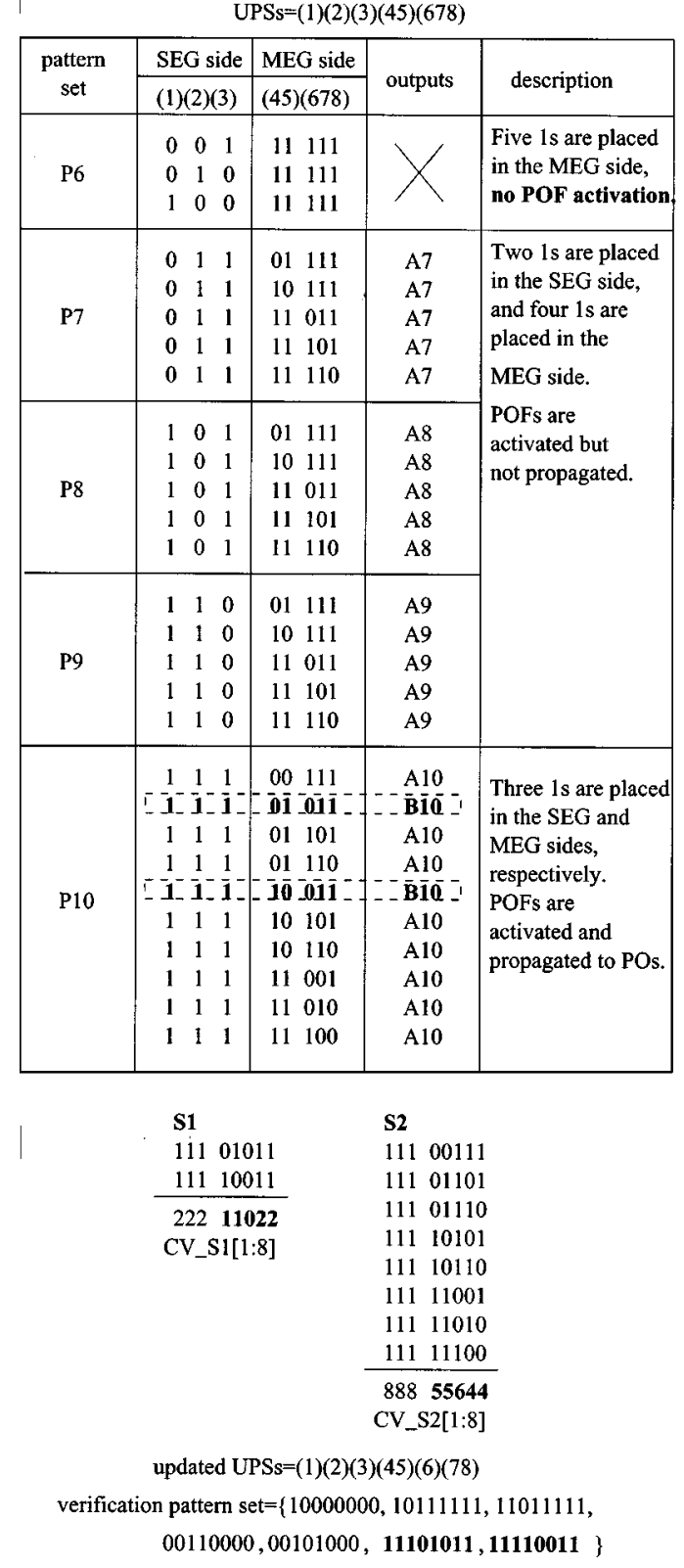

Fig. 17. The simulation results of $\Theta_{6}^{8}$ patterns.

Lemma 2: Given two pattern sets $S$ and $S^{\prime}$, if $C V \_S \neq$ $C V \_S^{\prime}$, then $S \neq S^{\prime}$.

Theorem 3: A pattern set $S$ turns to another pattern set $S^{\prime}$ after the port misplacements $\pi$. If $C V_{-} S \neq C V_{-} S^{\prime}$, then the port misplacements $\pi$ will be detected by $S$ patterns.

Proof: Because $C V \_S \neq C V_{-} S^{\prime}$, according to Lemma 2, $S \neq S^{\prime}$. Furthermore, because $|S|=\left|S^{\prime}\right|, S^{\prime}$ must exist a pattern $T \notin S$. Since $S$ consists of all patterns with the same outputs, the outputs of $T$ must be different with that of patterns in the $S$. Thus, the port misplacements $\pi$ will cause the outputs of $S$ and $S^{\prime}$ different and will be detected by $S$ patterns. Q.E.D.

According to Theorem 3, if $S 1$ in Fig. 17 is chosen as the verification patterns, the port misplacements that change $C V \_S 1[4: 8]$ will be detected. Consequently, the port misplacements which cannot change the $C V_{-} S 1[4: 8]$ are regarded as the remaining UPSs. The $C V_{-} S 1[4: 8]=11022$ is further grouped into subgroups, $(11)(0)(22)$ and it corre-
UPSs $=(1)(2)(3)(6)(45)(78)$

\begin{tabular}{|c|c|c|c|c|}
\hline \multirow{2}{*}{$\begin{array}{c}\text { pattern } \\
\text { set }\end{array}$} & SEG side & MEG side & \multirow{2}{*}{ outputs } & \multirow{2}{*}{ description } \\
\hline & $(1)(2)(3)(6)$ & $(45)(78)$ & & \\
\hline P11 & $\begin{array}{llll}1 & 1 & 1 & 0 \\
1 & 1 & 0 & 1 \\
1 & 0 & 1 & 1 \\
0 & 1 & 1 & 1 \\
\end{array}$ & $\begin{array}{ll}00 & 00 \\
00 & 00 \\
00 & 00 \\
00 & 00 \\
\end{array}$ & & $\begin{array}{l}\text { Three } 1 \mathrm{~s} \text { are placed } \\
\text { in the SEG side, } \\
\text { no POF activation. }\end{array}$ \\
\hline P12 & $\begin{array}{ccccc}\mathbf{1} & \mathbf{1} & \mathbf{1} & \mathbf{0} \\
1 & 1 & 0 & 0 \\
1 & 1 & 0 & 0 \\
1 & 1 & 0 & 0\end{array}$ & $\begin{array}{rl}-\overline{\mathbf{1 0}} & \overline{\mathbf{0}} \mathbf{0}^{-} \\
01 & 00 \\
00 & 10 \\
00 & 01\end{array}$ & $\begin{array}{c}-\overline{\mathbf{A}} \overline{2}-\overline{1} \\
\mathrm{~B} 12 \\
\mathrm{~B} 12 \\
\mathrm{~B} 12\end{array}$ & $\begin{array}{l}(45)(78) \cap(4)(578) \\
=(4)(5)(78) \\
(1)(2)(3)(6)+(4)(5)(78) \\
=(1)(2)(3)(4)(5)(6)(78)\end{array}$ \\
\hline P13 & $\begin{array}{llll}1 & 0 & 1 & 0 \\
1 & 0 & 1 & 0 \\
1 & 0 & 1 & 0 \\
1 & 0 & 1 & 0\end{array}$ & $\begin{array}{ll}10 & 00 \\
01 & 00 \\
00 & 10 \\
00 & 01\end{array}$ & $\begin{array}{l}\text { A13 } \\
\text { B13 } \\
\text { A13 } \\
\text { A13 }\end{array}$ & $\begin{array}{l}(4)(5)(78) \cap(5)(478) \\
=(4)(5)(78) \\
(1)(2)(3)(6)+(4)(5)(78) \\
=(1)(2)(3)(4)(5)(6)(78)\end{array}$ \\
\hline P14 & \begin{tabular}{cccc}
1 & 0 & 0 & 1 \\
1 & 0 & 0 & 1 \\
1 & 0 & 0 & 1 \\
\hdashline $\mathbf{1}$ & $\mathbf{0}_{-}^{-}$ & $\overline{0}_{-}$ & $\mathbf{1}$
\end{tabular} & {$\left[\begin{array}{rr}10 & 00 \\
01 & 00 \\
00 & 10 \\
-00 & 0 \\
\mathbf{0} & -\end{array}\right.$} & $\begin{array}{r}\mathrm{A} 14 \\
\mathrm{~A} 14 \\
\mathrm{~A} 14 \\
-\overline{\mathrm{B}} \overline{\mathbf{1}}-\overline{-}\end{array}$ & $\begin{array}{l}(4)(5)(78) \cap(457)(8) \\
=(4)(5)(7)(8) \\
(1)(2)(3)(6)+(4)(5)(7)(8) \\
=(1)(2)(3)(4)(5)(6)(7)(8)\end{array}$ \\
\hline
\end{tabular}

updated UPSs $=(1)(2)(3)(4)(5)(6)(7)(8)$

verification pattern set $=\{10000000,10111111,11011111,00110000$, $00101000,11101011,11110011,11001000,10010001\}$

Fig. 18. The simulation results of $\Theta_{3}^{8}$ patterns.

sponds to (45)(6)(78) of the UPSs. The port misplacements occurred in a subgroup will keep the CV remaining the same. Thus, when S1 is added into the verification pattern set, the updated UPSs become (1)(2)(3)(45)(6)(78). It is rewritten as $(1)(2)(3)(6)(45)(78)$ for convenience. For the S2, since the corresponding UPSs of the grouping result of $C V_{-} S 2[4: 8]$, (45)(6)(78), is the same as that of $C V_{-} S 1[4: 8]$, it is not added into the verification pattern set.

Fig. 18 shows the succeeding procedure that applies $\Theta_{3}^{8}$ into the core. In the P12, the result of the input port numbers grouping is (4)(578) when the pattern 11001000 is added into the verification pattern set. It intersects with the UPSs in the MEG side, (45)(78), to get the updated UPSs (4)(5)(78). Then it concatenates with the UPSs in the SEG side to form the updated UPSs $(1)(2)(3)(4)(5)(6)(78)$. This is shown in the description column in Fig. 18. In the P13, although the output of the pattern, 10100100, is different from that of the other patterns, it is not added into the verification pattern set. This is because the UPSs are not further reduced. In the P14, the pattern 10010001 reduces the UPSs from $(1)(2)(3)(4)(5)(6)(78)$ to $(1)(2)(3)(4)(5)(6)(7)(8)$ and the algorithm is terminated at this step. Because the generation of these pattern sets are from the smallest size to the largest size, other pattern sets with larger size in $\Theta_{3}^{8}$ are not generated and not shown in Fig. 18. The quality of the verification pattern set is determined by the measurement of fault coverage. The fault coverage is defined as

fault coverage $=$

$$
1 \text { - (\#_of_undetected_POFs/\#_of_all_POFs) }
$$

therefore, in this example, the fault coverage is 1 $0 /(8 !-1)=100 \%$ and the verification pattern set is 


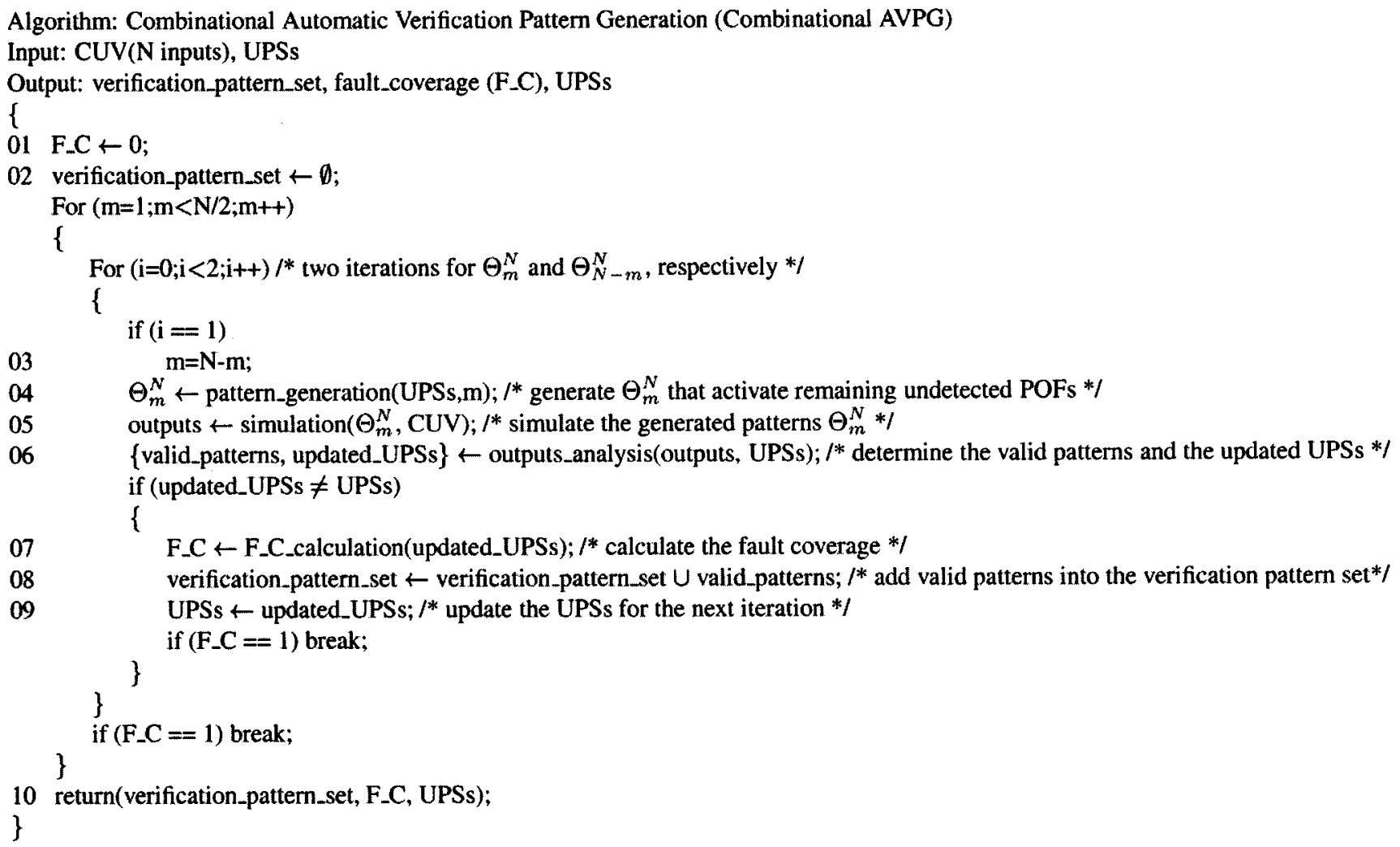

Fig. 19. The pseudocode of the combinational AVPG.

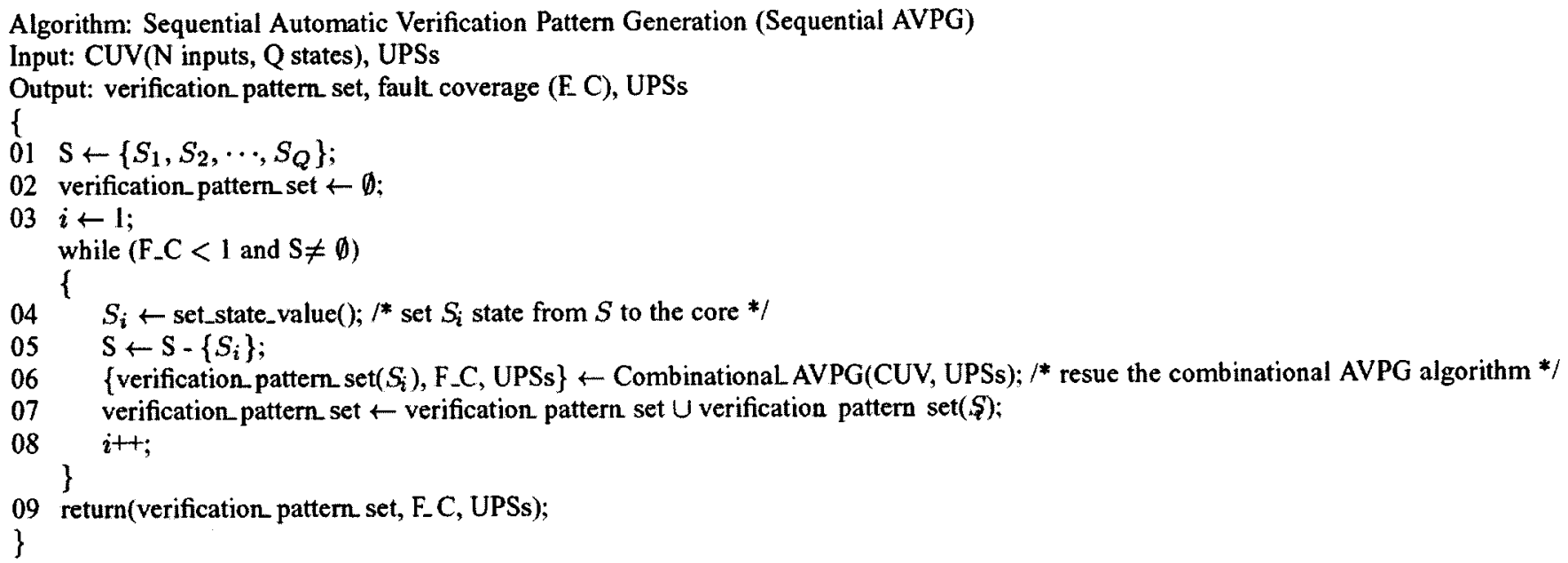

Fig. 20. The pseudocode of the sequential AVPG.

$\{10000000,10111111,11011111,00110000,00101000$, 11101011, 11110011, 11001000, 10010001$\}$.

Since the AVPG will generate all $\Theta_{m}^{N}$, for $m=1,2, \ldots, N-$ 1 if necessary and simulate the outputs for searching the verification patterns, it is a complete algorithm, i.e., given enough time, the verification pattern set for $100 \%$ fault coverage will be obtained. The pseudocode of this combinational AVPG is shown in Fig. 19. In line 4, 5, it generates patterns and simulates the outputs. The effectiveness of the simulated patterns is determined in line 6 . In line 8 , it adds the valid patterns into the verification pattern set. At the end of the algorithm, the verification pattern set, fault coverage, and UPSs are returned.

\section{B. The Sequential AVPG}

The development of the sequential AVPG is based on the same assumption as the combinational AVPG is, i.e., the CUV is preverified and fault free. The fault occurs only at the interconnection between the cores. Because the core is surrounded by the IEEE P1500 wrapper, by taking the advantages of the scan chains in the wrapper, the CUV can be set in arbitrary state_values. Hence, a sequential core can be seen as a combinational one and the verification pattern generation algorithm used in the combinational AVPG is applicable to the sequential AVPG. The only difference is that the sequential core has to be set a state_value before being evaluated. Different state_values 


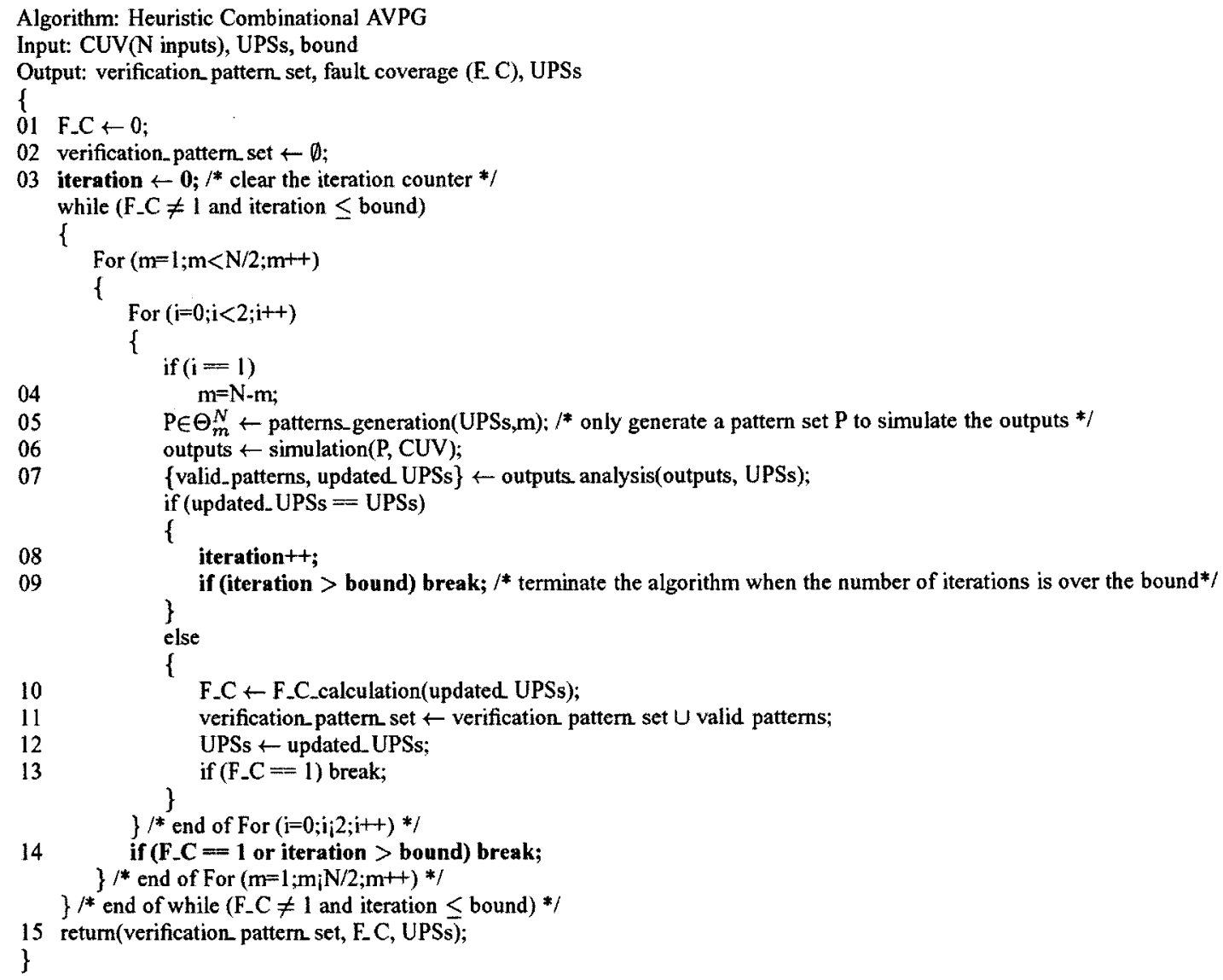

Fig. 21. The pseudocode of the heuristic combinational AVPG.

affect whether the fault effects could be propagated to POs or not. The algorithm sets the sequential core to every possible state until the fault coverage is $100 \%$. The pseudocode of the sequential AVPG is shown in Fig. 20. In line 4, it sets $S_{i}$ state to the sequential core before simulation. Then it reuses the combinational AVPG algorithm shown in Fig. 19. This process is listed in line 6 . The state_value has to be attached to the verification patterns obtained by the reused combinational AVPG. Thus, in line 6 , it uses verification_pattern_set $\left(S_{i}\right)$ to represent the verification pattern set obtained in $S_{i}$ state.

\section{The Heuristic AVPG}

Definition 12: An untestable POF is a POF which cannot be detected by $\Phi^{N}$.

The untestable POF is harmless for the integration, therefore, they should be regarded as detected POF in computing fault coverage.

The complete AVPG algorithm may not be practical in generating the verification pattern set for large designs. The possibility of existing untestable POFs in the CUV makes the algorithm very time-consuming. Therefore, a heuristic AVPG algorithm is proposed to trade-off between the fault coverage and the execution time. Here, we only address the heuristic combinational AVPG. This is because the combinational AVPG is the basis of the sequential AVPG.

We review Fig. 16, which shows the simulation outputs of applying $\Theta_{2}^{8}$ in the complete combinational AVPG. In this figure, the pattern sets P2 $\sim$ P5 are generated and simulated. However,
TABLE I

EXPERIMENTAL RESULTS OF THE HEURISTIC COMBINATIONAL AVPG

\begin{tabular}{c|c|c|c|c|c|c|c}
\hline \multirow{2}{*}{ bench } & \multicolumn{3}{|c|}{ parameters } & \multicolumn{3}{c}{ combinational AVPG } \\
\cline { 2 - 8 } & $\mid \mathrm{PI}$ & $|\mathrm{PO}|$ & lits. & \#_of_POFs & pats. & F_C(\%) & time(sec.) \\
\hline$c 17$ & 5 & 2 & 12 & $5 !-1$ & 5 & 100 & $<1$ \\
$c 880$ & 60 & 26 & 703 & $60 !-1$ & 255 & 99.999999 & 75 \\
$c 1355$ & 41 & 32 & 1032 & $41 !-1$ & 64 & 100 & 13.4 \\
$c 1908$ & 33 & 25 & 1497 & $33 !-1$ & 51 & 100 & 42 \\
$c 432$ & 36 & 7 & 372 & $36 !-1$ & 38 & 100 & 4.6 \\
$c 499$ & 41 & 32 & 616 & $41 !-1$ & 33 & 100 & 8.3 \\
$c 3540$ & 50 & 22 & 2934 & $50 !-1$ & 158 & 100 & 921 \\
$c 5315$ & 178 & 123 & 4369 & $178 !-1$ & 371 & 100 & 931 \\
$c 2670$ & 233 & 140 & 2043 & $233 !-1$ & 547 & 99.999999 & 729 \\
$c 7552$ & 207 & 108 & 6098 & $207 !-1$ & 1427 & 99.999999 & 1811 \\
$c 6288$ & 32 & 32 & 4800 & $32 !-1$ & 30 & 99.999999 & 175 \\
des & 256 & 245 & 7412 & $256 !-1$ & 428 & 100 & 159 \\
alu4 & 14 & 8 & 1278 & $14 !-1$ & 22 & 100 & 2.8 \\
apex6 & 135 & 99 & 904 & $135 !-1$ & 234 & 99.999999 & 406 \\
$i 9$ & 88 & 63 & 1453 & $88 !-1$ & 139 & 100 & 24.7 \\
$i 8$ & 133 & 81 & 4626 & $133 !-1$ & 266 & 100 & 415 \\
$i 7$ & 199 & 67 & 1311 & $199 !-1$ & 292 & 100 & 103 \\
$i 6$ & 138 & 67 & 1037 & $138 !-1$ & 174 & 100 & 84 \\
$i 5$ & 133 & 66 & 556 & $133 !-1$ & 155 & 100 & 63 \\
duke2 & 22 & 29 & 1746 & $22 !-1$ & 74 & 100 & 83.4 \\
rot & 135 & 107 & 1424 & $135 !-1$ & 529 & 99.999999 & 204 \\
$x 1$ & 51 & 35 & 2141 & $51 !-1$ & 275 & 99.999999 & 34.1 \\
$x 3$ & 135 & 99 & 1816 & $135 !-1$ & 249 & 99.999999 & 171 \\
$x 4$ & 94 & 71 & 1040 & $94 !-1$ & 398 & 99.999999 & 69 \\
pair & 173 & 137 & 2667 & $173 !-1$ & 217 & 100 & 443 \\
\hline & & & & & &
\end{tabular}

according to Theorem 1, any one of them can activate all remaining POFs and has the possibility to reduce the size of the remaining UPSs. Hence, the heuristic approach is to generate 
TABLE II

EXPERIMENTAL RESULTS OF THE HEURISTIC SEQUENTIAL AVPG

\begin{tabular}{|c|c|c|c|c|c|c|c|c|}
\hline \multirow[b]{2}{*}{ bench } & \multicolumn{5}{|c|}{ parameters } & \multicolumn{3}{|c|}{ sequential AVPG } \\
\hline & $|\mathrm{PI}|$ & $|\mathrm{PO}|$ & lits. & FFs & \#_of_POFs & pats. & F_C $(\%)$ & time(sec.) \\
\hline$s 1196$ & 14 & 14 & 1009 & 18 & $14 !-1$ & 24 & 99.999999 & 16 \\
\hline$s 1238$ & 14 & 14 & 1041 & 18 & $14 !-1$ & 16 & 99.999999 & 27 \\
\hline$s 13207$ & 31 & 121 & 11241 & 669 & $31 !-1$ & 64 & 99.999999 & 1165 \\
\hline$s 1423$ & 17 & 5 & 1164 & 74 & $17 !-1$ & 107 & 100 & 48 \\
\hline$s 1488$ & 8 & 19 & 1387 & 6 & $8 !-1$ & 10 & 100 & 6 \\
\hline$s 1494$ & 8 & 19 & 1393 & 6 & $8 !-1$ & 14 & 100 & 5 \\
\hline$s 15850$ & 14 & 87 & 13659 & 597 & $14 !-1$ & 11 & 99.999999 & 256 \\
\hline$s 208$ & 11 & 2 & 166 & 8 & $11 !-1$ & 25 & 100 & 6 \\
\hline$s 27$ & 4 & 1 & 18 & 3 & $4 !-1$ & 3 & 100 & $<1$ \\
\hline$s 344$ & 9 & 11 & 269 & 15 & $9 !-1$ & 10 & 100 & 2 \\
\hline$s 349$ & 9 & 11 & 273 & 15 & $9 !-1$ & 10 & 100 & 2 \\
\hline$s 35932$ & 35 & 320 & 28269 & 1728 & $35 !-1$ & 549 & 99.999999 & 3113 \\
\hline$s 420$ & 19 & 2 & 336 & 16 & $19 !-1$ & 294 & 99.999999 & 16 \\
\hline$s 510$ & 19 & 7 & 424 & 6 & $19 !-1$ & 170 & 100 & 16 \\
\hline$s 5378$ & 35 & 49 & 4212 & 164 & $35 !-1$ & 393 & 99.999999 & 363 \\
\hline$s 641$ & 35 & 23 & 539 & 19 & $35 !-1$ & 86 & 99.999999 & 23 \\
\hline$s 713$ & 35 & 23 & 591 & 19 & $35 !-1$ & 70 & 99.999999 & 20 \\
\hline$s 820$ & 18 & 19 & 757 & 5 & $18 !-1$ & 117 & 99.999999 & 43 \\
\hline$s 832$ & 18 & 19 & 767 & 5 & $18 !-1$ & 23 & 100 & 9 \\
\hline$s 838$ & 35 & 2 & 670 & 32 & $35 !-1$ & 1845 & 99.999999 & 279 \\
\hline$s 9234$ & 19 & 22 & 7971 & 228 & $19 !-1$ & 23 & 100 & 109 \\
\hline$s 953$ & 16 & 23 & 743 & 29 & $16 !-1$ & 95 & 100 & 16 \\
\hline$s c f$ & 27 & 56 & 1865 & 7 & $27 !-1$ & 417 & 100 & 84 \\
\hline $\min \max 10$ & 13 & 10 & 735 & 30 & $13 !-1$ & 51 & 100 & 24 \\
\hline $\min \max 12$ & 15 & 12 & 909 & 36 & $15 !-1$ & 71 & 100 & 21 \\
\hline $\min \max 32$ & 35 & 32 & 3089 & 96 & $35 !-1$ & 706 & 99.999999 & 154 \\
\hline $\min \max 5$ & 8 & 5 & 335 & 15 & $8 !-1$ & 10 & 100 & 2 \\
\hline mult32 & 32 & 64 & 14745 & 32 & $32 !-1$ & 31 & 100 & 20 \\
\hline$s b c$ & 40 & 56 & 1586 & 28 & $40 !-1$ & 182 & 100 & 91 \\
\hline$t l c$ & 3 & 5 & 423 & 10 & $3 !-1$ & 3 & 100 & $<1$ \\
\hline
\end{tabular}

arbitrary one of them to simulate the outputs in one iteration instead of all pattern sets. For example, it can only generate and simulate $\mathrm{P} 2$ or P3, etc. No matter what the result of the simulation of generated pattern set is, it proceeds to the next iteration. On the other hand, the heuristic AVPG generates the verification patterns iteratively, therefore, it sets an iteration counter to bound the processing time.

The heuristic combinational AVPG algorithm is shown in Fig. 21. In line 5, it generates a pattern set $P$ instead of all $\Theta_{m}^{N}$ heuristically where $P$ is a subset of $\Theta_{m}^{N}$. The pattern set $P$ satisfies the requirement of activating all remaining undetected POFs. The pattern set P3, P8, or P12 listed in Figs. 16-18 are all instances of $P$. The check points in the algorithm bound the AVPG to be executed in the acceptable run time. These codes in lines 3, 8, 9, and 14 are all highlighted in Fig. 21.

\section{EXPERIMENTAL RESULTS}

Rather than simulating the entire SoC, the complexity of SoC design verification can be significantly alleviated by using preverified IP cores and concentrating on verifying the integration of the cores in the SoC. The interconnection verification among each single block during integration is the first step of the core-based SoC design verification. Furthermore, the generation of the verification patterns for verifying the interconnection among the cores only depends on the functionality of the added core. Therefore, the experiment only reports the results of single block examples.

The heuristic POF AVPG algorithm described above has been integrated into the SIS [8] environment, which is developed by the University of California at Berkeley. Experiments are conducted over a set of ISCAS-85 and MCNC combinational benchmarks for the heuristic combinational AVPG and a set of ISCAS-89 sequential benchmarks for the heuristic sequential AVPG. Note that the benchmarks are in Berkeley Logic Interchange Format (BLIF) format which is a netlist-level design description. However, only the simulation information of these benchmarks are used to conduct the experiments and therefore, arbitrary level of design description can be used for generating verification patterns. Table I summaries the experimental results of the heuristic combinational AVPG. The first five columns show the parameters of each benchmark, including name, |PI|, $|\mathrm{PO}|$, the number of literals (lits.), and the number of POFs. The $|\mathrm{PI}|$ represents the number of inputs and the size of the POFs set is $|\mathrm{PI}| !-1$. The $|\mathrm{PO}|$ represents the number of outputs and influences on the probability of fault effect propagation. The number of literals indicates the complexity of a benchmark. The remaining columns show the number of verification patterns (pats.), fault coverage (F_C), and CPU time (time). The iteration bound was set to 100 . The CPU time is measured on an Ultra Sparc II workstation. The algorithm will be terminated automatically if the iteration counter is over the bound or the fault coverage reaches $100 \%$ and the verification pattern set, fault coverage, and updated UPSs are returned. According to Table I, the fault coverage of more than half benchmarks achieve $100 \%$ and the processing time is acceptable. Furthermore, the size of the verification pattern sets are very small as compared with the $(N !-1)$ POFs. For example, the number of POFs in c5315 is $(178 !-1)$, but the size of the verification pattern set is only 371 
for $100 \%$ fault coverage. For the other benchmarks, the fault coverage also reach $99.999999 \%$ high.

The results demonstrate that the heuristic combinational AVPG is very efficient to generate high-quality verification pattern set. Note that the undetected POFs in a benchmark could be untestable. If we omit these untestable POFs from the fault set, the fault coverage of the pattern set would be even higher.

Table II summaries the experimental results of the heuristic sequential AVPG. The fifth column lists the number of flip-flops in a benchmark. Table II also demonstrates that all sequential benchmarks achieve high fault coverage with acceptable run time similar to the results indicated in the heuristic combinational AVPG.

\section{CONCLUSION}

In the SoC era, the embedded cores are mixed and integrated to create a system chip. System designers integrate those cores manually and have the possibility of incorrect integration due to the misplaced I/O ports. Furthermore, without the knowledge of the internal structures of the embedded cores, system designers have difficult time to locate the position of having erroneous interconnection. Therefore, we adopt the connectivity-based POF model and use the proposed verification mechanism to integrate the system blockwise. This raised abstraction level of the design verification reduce the time on functional verification in core-based design methodology.

The POF-based AVPG algorithm generates the verification pattern set for $(N !-1)$ POFs systematically by using the property of the POF activation and domination and the implicit UPS's representation. We exploit the IEEE P1500 wrapper structure as the integration verification mechanism. The POF verification provides a sufficient high level of confidence on verifying the correctness of the core-based system design.

\section{REFERENCES}

[1] S.-W. Tung and J.-Y. Jou, "Verification pattern generation for core-based design using port-order fault model," in Proc. Asian Test Symp., Dec. 1998, pp. 402-407.

[2] H. Chang, L. Cooke, M. Hunt, G. Martin, A. McNelly, and L. Todd, Surviving the SoC Revolution-A Guide to Platform-Based Design. Norwell, MA: Kluwer Academic, 1999.

[3] J. Bergeron, Writing Testbenches-Functional Verification of $H D L$ Model. Norwell, MA: Kluwer Academic, 2000.

[4] P. Goel and M. T. McMahon, "Electronic chip-in-place test," in Proc. IEEE Int. Test Conf., Oct. 1982, pp. 83-90.

[5] A. Hassan, V. K. Agarwal, B. Nadeau-Dostie, and J. Rajski, "BIST of PCB interconnects using boundary-scan architecture," IEEE Trans. Computer-Aided Design, vol. 11, pp. 1278-1288, Oct. 1992.

[6] J. A. Rowson and A. Sangiovanni-Vincentelli, "Interface-based design," in Proc. Design Automation Conf., June 1997, pp. 178-183.

[7] S.-W. Tung and J.-Y. Jou, "A logic fault model for library coherence checking," J. Inform. Sci. Eng., pp. 567-586, Sept. 1998.

[8] E. M. Sentovich, K. T. Singh, C. Moon, H. Savoj, R. K. Brayton, and A. Sangiovanni-Vincentelli, "Sequential circuit design using synthesis and optimization," in Proc. IEEE Int. Conf. Computer Design, Oct. 1992, pp. $328-333$.

[9] E. J. Marinissen, Y.Zorian, R. Kapur, T. Taylor, and L. Whetsel, “Toward a standard for embedded core test: An example," in Proc. IEEE Int. Test Conf., Sept. 1999, pp. 616-627.
[10] M. Abramovici, M. A. Breuer, and A. D. Friedman, Digital Systems Testing and Testable Design. New York: Computer Science Press, 1990, p. 95.

[11] Y. Zorian, E. J. Marinissen, and S. Dey, "Testing embedded-core-based system chips," in Proc. IEEE Int. Test Conf., Oct. 1998, pp. 130-143.

[12] S. Runyon, "Testing big chips becomes an internal affair," IEEE Spectrum Mag., vol. 36, pp. 49-55, Apr. 1999.

[13] R. G. B. Bennetts. IEEE P1500 Embedded Core Test Standard. [Online]. Available: http://www.semiconductorfabtech.com/dft/tutorial/p1500.PDF

[14] M. Ricchetti. Overview of proposed ieee scalable architecture for testing embedded cores. presented at IEEE P1500 SECT Meeting During DAC'01. [Online]. Available: http://www.grouper. ieee.org/groups/1500/dac01/ctag-dac01.pdf.

[15] K.-T. Cheng and J.-Y. Jou, "A functional fault model for sequential machines," IEEE Trans. Computer-Aided Design, vol. 11, pp. 1065-1073, Sept. 1992.

[16] M. H. Schulz, E. Trischler, and T. M. Sarfert, "SOCRATES: A highly efficient automatic test pattern generation system," IEEE Trans. Computer-Aided Design, vol. 7, pp. 126-137, Jan. 1988.

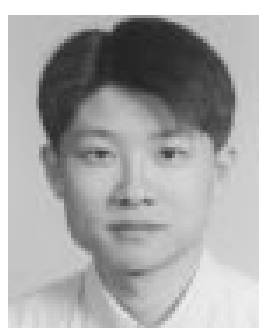

Chun-Yao Wang (S'00) was born on Oct 21, 1973 in Taiwan, ROC. He graduated from the Department of Electronics Engineering, National Taipei Institute of Technology, Taiwan, ROC, in 1994. He is currently pursuing the Ph.D. degree at the Department of Electronics Engineering, National Chiao Tung University, Hsinchu, Taiwan, ROC.

His research interests include testing, design verification, and logic synthesis.

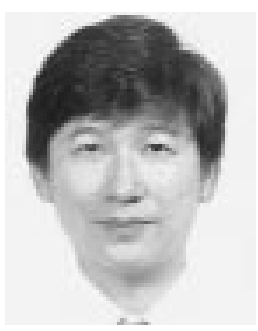

Shing-Wu Tung (M'92) received the B.S. degree from the Department of Electrical Engineering, National Cheng Kung University, and the M.S. degree from the Department of Computer Science and Information Engineering at National Chiao Tung University, Hsinchu, Taiwan, ROC, in 1987 and 1989 , respectively. He is working toward the Ph.D. degree at the Department of Electronics Engineering, National Chiao Tung University.

He is a senior manager at Prolific Technology Inc. He has worked at Industrial Technology Research Institute. His current research activities focus on design verification of platformbased SoC design. His other areas of interest include computer architecture, CPU design, and VLSI system verification.

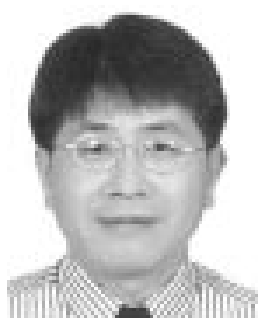

Jing-Yang Jou (S'82-M'83-SM'02) received the B.S. degree from the Department of Electrical Engineering at National Taiwan University, Taiwan, ROC, and the M.S. and Ph.D. degrees from the Department of Computer Science, University of Illinois at Urbana-Champaign, in 1979, 1983, and 1985 , respectively.

$\mathrm{He}$ is currently a full Professor and Chairman of Electronics Engineering Department at National Chiao Tung University, Hsinchu, Taiwan, ROC. Before joining National Chiao Tung University, he was with GTE Laboratories and AT\&T Bell Laboratories. His research interests include behavioral, logic and physical synthesis, design verification, and CAD for low power. He has published more than 100 journal and conference papers.

Dr. Jou is a member of Tau Beta Pi and is the recipient of the distinguished paper award of the IEEE International Conference on Computer-Aided Design, 1990. He served as the Technical Program Chair of the Asia-Pacific Conference on Hardware Description Languages (APCHDL'97). 